\title{
A facile synthesis, and antimicrobial and anticancer activities of some pyridines, thioamides, thiazole, urea, quinazoline, $\beta$-naphthyl carbamate, and pyrano[2,3- $d]$ thiazole derivatives
}

\author{
Yasser H. Zaki ${ }^{1,2^{*}}$, Marwa S. Al-Gendey ${ }^{3}$ and Abdou O. Abdelhamid ${ }^{4}$
}

\begin{abstract}
Background: Chalcones have a place with the flavonoid family and show a few very important pharmacological activities. They can used as initial compounds for synthesis of several heterocyclic compounds. The compounds with the backbone of chalcones have been reported to possess various biological activities.

Results: Pyridine and thioamide derivatives were obtained from the reaction of 3-(furan-2-yl)-1-(p-tolyl)prop-2-en1 -one with the appropriate amount of malononitrile, benzoylacetonitrile, ethyl cyanoacetate and thiosemicarbazide in the presence of ammonium acetate. The reaction of 3,5-di(furan-2-yl)-4,5-dihydro-1 H-pyrazole-1-carbothioamide with ethyl 2-chloro-3-oxobutanoate, 3-chloropentane-2,4-dione or ethyl chloroacetate produced thiazole derivatives. Pyrano[2,3- $d$ ] thiazole derivatives were obtained as well from thiazolone to arylidene malononitrile. The structures of the title compounds were clarified by elemental analyses, and FTIR, MS and NMR spectra. Some compounds were screened against various microorganisms (i.e., bacteria +ve, bacteria - ve and fungi). We observed that compounds (3a), (4a), (4d), (5), (7) and compound (8) exhibited high cytotoxicity against the MCF-7 cell line, with IC 50 values of $23.6,13.5,15.1,9.56,14.2$ and $23.5 \mu \mathrm{mol} \mathrm{mL}^{-1}$, respectively, while compound (9) was displayed the lowest values against MCF-7 cell lines.

Conclusions: Efficient synthetic routes for some prepared pyridines, pyrazoline, thioamide, thiazoles and pyrano[2,3d]thiazole were created. Moreover, selected newly-synthesized products were evaluated for their antitumor activity against two carcinoma cell lines: breast MCF-7 and colon HCT-116 human cancer cell lines.
\end{abstract}

Keywords: Antimicrobial, Anticancer, Pyridines, Thioamides, Thiazoles, Pyrano[2,3-d]thiazoles

\section{Background}

The chalcones (1,3-diaryl-2-propenones) and their derivatives are important intermediates in organic synthesis [1-3]. They serve as starting material for the synthesis of a variety of heterocyclic compounds of physiological importance. Due to the presence of

\footnotetext{
*Correspondence: yzaki2002@yahoo.com

${ }^{1}$ Department of Chemistry, Faculty of Science, Beni-Suef University, Beni-Suef 62514, Egypt

Full list of author information is available at the end of the article
}

enone functionality in chalcone, moiety confers antimicrobial [4-6], anti-inflammatory [7], antimalarial $[8,9]$, antileishmanial [10], antioxidant [11], antitubercular [12, 13], anticancer [14, 15] and other biological activities. In addition, thiazoles are involved in development of drugs for the treatment of allergies [16], hypertension [17], inflammation [18], schizophrenia [19], bacterial infections [20], HIV [21], sleep disorders [22] and, most recently, for of pain [23]. They function as fibrinogen receptor antagonists with antithrombotic activity [24], and as new inhibitors of bacterial DNA 
gyrase B [25]. In addition, pyrano[2,3- $d]$ thiazoles are biologically interesting compounds with diabetes, obesity, hyperlipidemia, and atherosclerotic diseases [26]. They are also known to show antimicrobial, bactericidal, fungicidal and molluscicidal activities [27, 28]. In continuation of our previous work on the synthesis of new anticancer agents [29-34], we present here efficient syntheses of novel pyridines, pyrazolines, thiazoles and pyrano[2,3-d]thiazole derivatives which have not been previously reported. We investigated the anticarcinogenic effects against MCF-7, and the antibacterial activity of HCT-116 on human cancer cell lines against Streptococcus pneumonia and Bacillus subtilis as examples of Gram-positive bacteria and Pseudomonas aeruginosa and Escherichia coli as examples of Gram-negative bacteria.

\section{Results and discussion}

\section{Chemistry}

Reactions of 3-(furan-2-yl)-1-( $p$-tolyl)prop-2-en-1-one (1a) with an appropriate amount of malononitrile, benzoylacetonitrile, ethyl cyanoacetate, and thiosemicarbazide yielded 2-amino-4-(furan-2-yl)-6-( $p$-tolyl)nicotinonitrile (2a), 4-(furan-2-yl)-2-phenyl-6-( $p$-tolyl)nicotine-nitrile (3a), 4-(furan-2-yl)-2-oxo-6-( $p$-tolyl)-1,2-dihydropyridine-3-carbonitrile (4a), and 3,5-di(furan-2-yl)-4,5-dihydro- $1 H$-pyrazole-1-carbothioamide (5), respectively (Scheme 1). Structures $\mathbf{2 a}-\mathbf{4 a}$ and $\mathbf{5}$ were elucidated on the basis of elemental analyses and spectral data.

Analogy, heating of the appropriate chalcone (1b-f) with malononitrile, benzoylacetonitrile, or ethyl cyanoacetate in glacial acetic acid in the presence of ammonium acetate created pyridine derivatives $(\mathbf{2}-\mathbf{4}) \mathbf{b}-\mathbf{f}(c f$. Scheme 1). Structures (2-4)b-f were elucidated by elemental analysis and spectral data (cf. "Experimental"). On the other hand, a reaction of 3,5-di(furan-2-yl)-4,5-dihydro- $1 H$-pyrazole-1-carbothioamide (5), which was prepared from 1e to thiosemicarbazide (each with ethyl 2-chloro-3-oxobutanoate, 3-chloropentane-2,4-dione, or ethyl 2-chloroacetate in ethanolic triethylamine) afforded ethyl 2-(3,5-di(furan-2-yl)-4,5-dihydro-1 $H$-pyrazol-1-yl)4-methylthiazole-5-carboxylate (6), 1-(2-(3,5-di(furan2-yl)-4,5-dihydro-1 $H$-pyrazol-1-yl)-4-methylthiazol-5-yl) ethan-1-one (7), and 2-(3,5-di(furan-2-yl)-4,5-dihydro$1 H$-pyrazol-1-yl)thiazol-4(5H)-one (8), respectively (Scheme 2). Structures (6-8) were confirmed with elemental analysis, spectral data, and chemical transformation.

Compound (6) was further reacted with hydrazine hydrate afforded 2-(3,5-di(furan-2-yl)-4,5-dihydro$1 H$-pyrazol-1-yl)-4-methylthiazole-5-carbohydrazide (9) (Scheme 3). Structure 9 was elucidated by elemental analysis, spectra and chemical transformations.
Thus, compound 9 reacted with nitrous acid yielded 2-(3,5-di(furan-2-yl)-4,5-dihydro- $1 H$-pyrazol-1-yl)4-methylthiazole-5-carbonyl azide (10). Structure 10 was confirmed by elemental analyses, spectral data and chemical transformation.

Treatment of compound $\mathbf{1 0}$ with each of the appropriate amounts of aniline, 4-toluidine, or anthranilic acid in boiling dioxane yielded 1-(2-(3,5-di(furan-2-yl)-4,5-dihydro$1 H$-pyrazol-1-yl)-4-methylthiazol-5-yl)-3-phenylurea (11a), 1-(2-(3,5-di(furan-2-yl)-4,5-dihydro- $1 H$-pyrazol1-yl)-4-methylthiazol-5-yl)-3-(p-tolyl)urea (11b), and 3-(2-(3,5-di(furan-2-yl)-4,5-dihydro- $1 H$-pyrazol-1-yl)4-methylthiazol-5-yl)quinazoline-2,4(1H, 3H)-dione (12), respectively. Additionally, compound 10 reacted with 2-naphthol in boiling benzene afforded naphthalen2-yl(2-(3,5-di(furan-2-yl)-4,5-dihydro- $1 H$-pyrazol-1-yl)4-methylthiazol-5-yl)carbamate (13) (Scheme 3). The structure of compound $\mathbf{1 2}$ was confirmed by elemental analyses, spectral data, and an alternative synthetic route. Thus, compound $\mathbf{1 0}$ reacted with methyl anthranilate in dioxane afforded a product identical in all aspects $(\mathrm{mp}$, mixed $\mathrm{mp}$, and spectra) to compound $\mathbf{1 2}$.

Finally, treatment of compound 8 with benzylidenemalononitrile (14a) in refluxing ethanol containing a catalytic amount of piperidine afforded 5-amino-2(3,5-di(furan-2-yl)-4,5-dihydro- $1 H$-pyrazol-1-yl)-7phenyl-7H-pyrano[2,3- $d$ ] thiazole-6-carbonitrile (15a) (Scheme 4). The structure of (15a) was elucidated by elemental analysis, spectral data, and a synthetic route. Furthermore, the infrared (IR) spectrum showed bands at $3388-3280 \mathrm{~cm}^{-1}$, which corresponded to the $\left(\mathrm{NH}_{2}\right)$ group. Thus, a mixture of malononitrile, benzaldehyde, and 2-(3,5-di(furan-2-yl)-4,5-dihydro-1 $H$-pyrazol-1-yl) thiazol-4 $(5 H)$-one $(8)$ in ethanol containing a few drops of piperidine as a catalyst heated under reflux afforded a product identical in all aspects $(\mathrm{mp}$, mixed $\mathrm{mp}$, and spectra) with (15a). Similarly, compound 8 reacted with 14b afforded 5-amino-2-(3,5-di(furan-2-yl)-4,5-dihydro$1 H$-pyrazol-1-yl)-7-(p-tolyl)-7H-pyrano[2,3-d] thiazole6-carbonitrile (15b) (Scheme 4).

\section{Cytotoxicity evaluations}

The in vitro growth inhibitory activity of the synthesized compounds 3a, 4a, 4d-4f, 5, 7, 8, 9, 11a, and 11b was investigated against two carcinoma cell lines: breast MCF-7 and colon HCT-116 human cancer cell lines in comparison with the Imatinib anticancer standard drug (cisplatin) under the same conditions using the crystal violet viability assay. Data generated were used to plot a dose response curve where the concentration of test compounds required to kill $50 \%$ of the cell population $\left(\mathrm{IC}_{50}\right)$ was determined and is summarized in Table 1 . The $\mathrm{IC}_{50}$ values of the synthesized compounds 


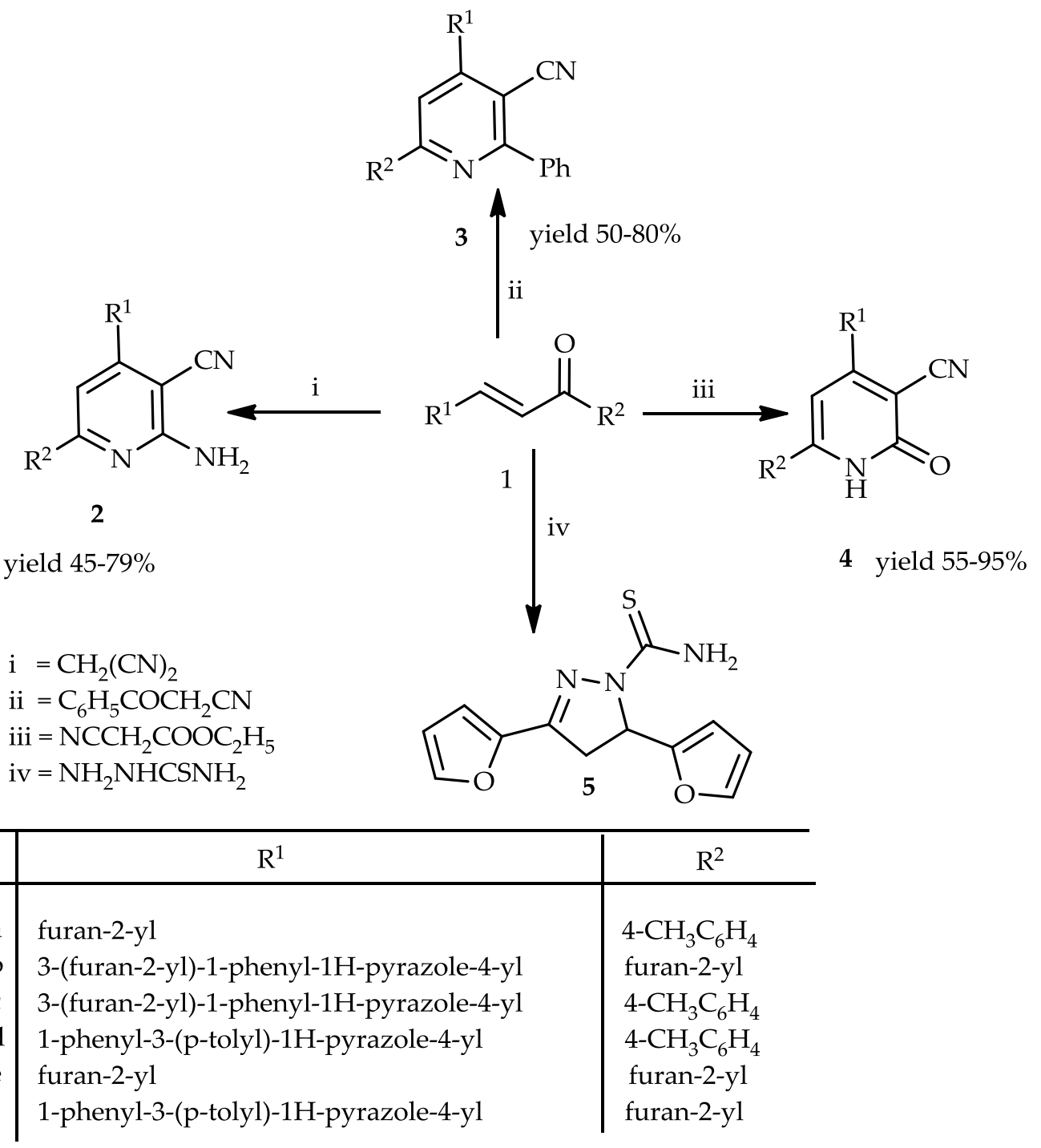

Scheme 1 Synthesis of pyridine derivatives (2-4) and thioamide (5)

4a, 4d, 5, 7, and 8, $\left(\mathrm{IC}_{50}=9.65-23.6 \mu \mathrm{mol} \mathrm{mL}^{-1}\right)$ were comparable to that of Imatinib. We observed that compounds 3a, 4a, 4d, 5, 7, and 8 exhibited high cytotoxicity against the MCF-7 cell line, with $\mathrm{IC}_{50}$ values of 23.6 , $13.5,15.1,9.56,14.2$ and $23.5 \mu \mathrm{mol} / \mathrm{mL}$, respectively, while compound 9 was observed as having the lowest against the MCF-7 cell lines. Our results showed that compounds $4 \mathrm{e}, 4 \mathrm{f}, 11 \mathrm{a}$ and $11 \mathbf{b}$ had the lowest $\mathrm{IC}_{50}$ values against HCT-116 cancer cells.

\section{Antimicrobial activity}

Nineteen of the newly synthesized target compounds were evaluated for their in vitro antibacterial activity against Streptococcus pneumonia and Bacillus subtilis (as examples of Gram-positive bacteria) and Pseudomonas aeruginosa and Escherichia coli (as examples of Gramnegative bacteria). They were also evaluated for their in vitro antifungal activity against a representative panel of fungal strains i.e., Aspergillus fumigatus and Candida albicans fungal strains. Ampicillin and Gentamicin are used as reference drugs for in vitro antibacterial activity while Amphotericin B is a reference drug for in vitro antifungal activity, respectively, at The Regional Center for Mycology and Biotechnology at Al-Azhar University (Nasr City, Cairo, Egypt). The results of testing for antimicrobial effects are summarized in Table 2.

\section{Experimental section}

\section{General information}

All melting points were measured with a Gallenkamp melting point apparatus (Weiss-Gallenkamp, London, 


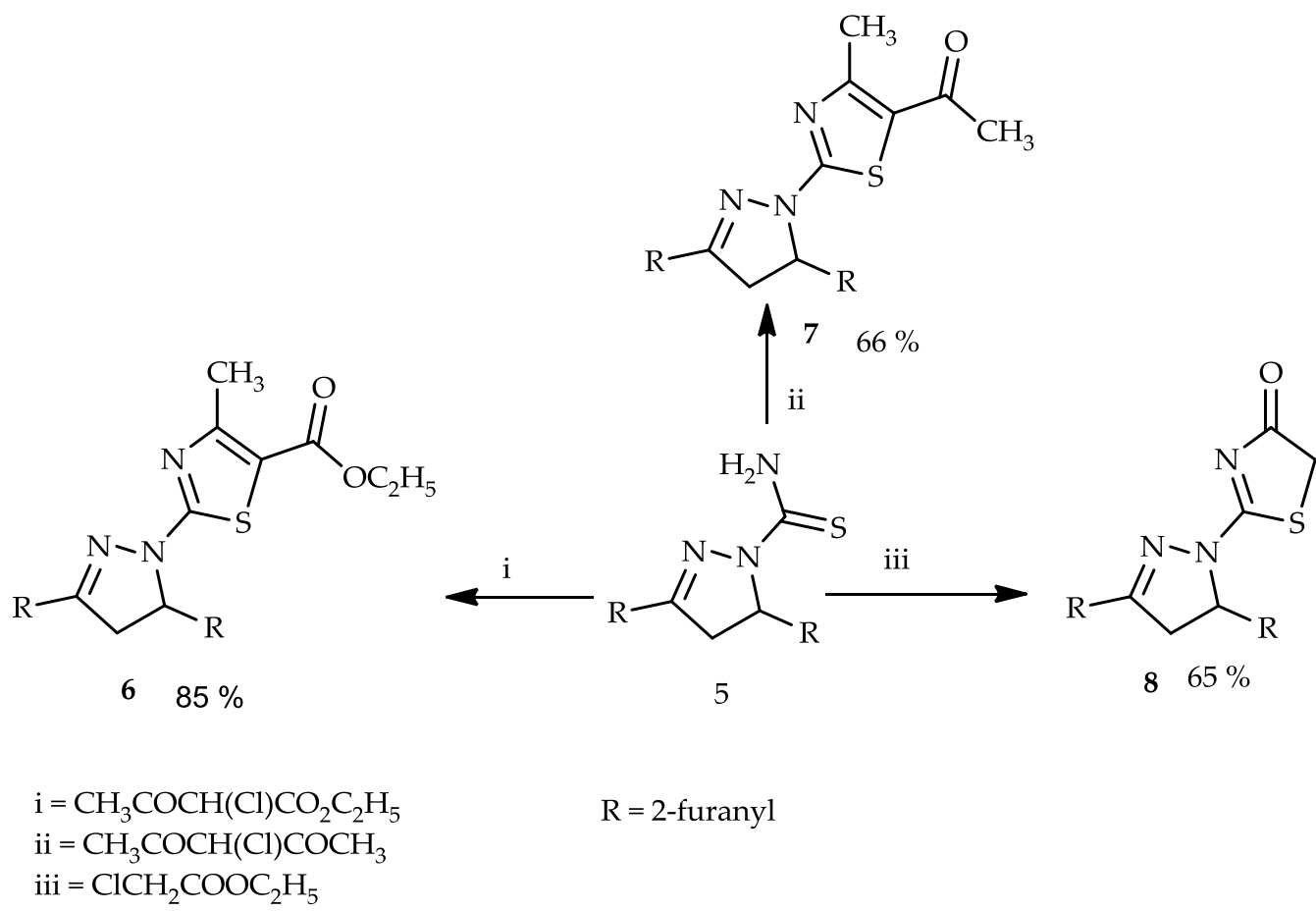

Scheme 2 Synthesis of thiazole derivatives 6-8

UK). The infrared spectra were recorded using potassium bromide disks on pye Uni-cam SP 3300 and Shimadzu FT-IR 8101 PC infrared spectrophotometers (Pye Unicam Ltd. Cambridge, England, and Shimadzu, Tokyo, Japan, respectively). The NMR spectra were recorded on a Varian Mercury VX-300 NMR spectrometer (Varian, Palo Alto, CA, USA). ${ }^{1} \mathrm{H}$ spectra were run at $300 \mathrm{MHz}$ and ${ }^{13} \mathrm{C}$ spectra were run at $75.46 \mathrm{MHz}$ in deuterated chloroform $\left(\mathrm{CDCl}_{3}\right)$ or dimethyl sulphoxide (DMSOd6). Chemical shifts were related to that of the solvent. Mass spectra were recorded on a Shimadzu GCMS-QP 1000 EX mass spectrometer (Shimadzu) at $70 \mathrm{eV}$. Elemental analyses were carried out at the Microanalytical Center of Cairo University. The antimicrobial and antcancer screening was performed at the Regional Center for Mycology and Biotechnology, Al-Azhar University, Cairo, Egypt.

\section{General methods for the synthesis of pyridines (2-4)a-f}

Method A A mixture of the appropriate chalcones (1a-f) $(10 \mathrm{mmol})$, and the appropriate amount of malononitrile, benzoylacetonitrile, or ethyl cyanoacetate $(10 \mathrm{mmol})$ in glacial acetic acid containing ammonium acetate $(0.77 \mathrm{~g}$, $10 \mathrm{mmol}$ ) was refluxed for $3-4 \mathrm{~h}$, and the acetic acid was evaporated under reduced pressure, left to cool, then poured. gradually with stirring onto crushed ice. The solid formed was filtered off, dried, and recrystallized from an appropriate solvent to obtain the corresponding pyridines (2-4)a-f, respectively.

Method B A mixture of the appropriate aldehydes $(10 \mathrm{mmol})$, arylketone $(10 \mathrm{mmol})$, and the appropriate amount of malononitrile, benzoylacetonitrile, or ethyl cyanoacetate $(10 \mathrm{mmol})$ in $n$-butanol $(20 \mathrm{~mL})$ containing ammonium acetate $(6.00 \mathrm{~g}, 77 \mathrm{mmol})$ was refluxed for 3-4 h, then the solvent evaporated under reduced pressure, left to cool, then poured gradually with stirring onto crushed ice. The solid formed was filtered off, dried, and recrystallized from an appropriate solvent to obtain products that were identical in all respects $(\mathrm{mp}$, mixed $\mathrm{mp}$, and IR spectra) with the corresponding pyridines (2-4)a-f, respectively. The products $(\mathbf{2}-\mathbf{4}) \mathbf{a}-\mathbf{f}$ together with their physical constants are listed below.

2-Amino-4-(furan-2-yl)-6-(p-tolyl)nicotinonitrile (2a) Pale yellow solid from glacial acetic acid, yield (1.79 g, 65\%), mp: $259-260^{\circ} \mathrm{C}$; IR $\left(\mathrm{KBr}, \mathrm{cm}^{-1}\right): 3304,3260$ $\left(\mathrm{NH}_{2}\right), 3145(=\mathrm{C}-\mathrm{H}), 2914(-\mathrm{C}-\mathrm{H}), 2208(-\mathrm{CN}), 1647$ $(-\mathrm{C}=\mathrm{N}) ;{ }^{1} \mathrm{H}$ NMR $\left(\mathrm{CDCl}_{3}\right): \delta 2.46\left(\mathrm{~s}, 3 \mathrm{H}, 4-\mathrm{CH}_{3} \mathrm{C}_{6} \mathrm{H}_{4}\right)$, $6.63(\mathrm{t}, 1 \mathrm{H}, J=4 \mathrm{~Hz}$, furan $\mathrm{H}-4), 7.17(\mathrm{~s}, 1 \mathrm{H}$, pyridine $\mathrm{H}-5), 7.22-7.25$ (m, 3H, ArH's and furan H-3), 7.40 (s, br., $\left.2 \mathrm{H}, \mathrm{NH}_{2}\right), 7.58-7.59(\mathrm{~d}, 1 \mathrm{H}, J=4 \mathrm{~Hz}$, furan $\mathrm{H}-5)$, 
<smiles>[R]C1=NN(c2nc(C)c(C(=O)OCC)s2)C([R])C1</smiles>

6<smiles>[Y11]CC[14CH2]NC(=O)Nc1sc(N2N=C([R])CC2[R])nc1C</smiles>

11

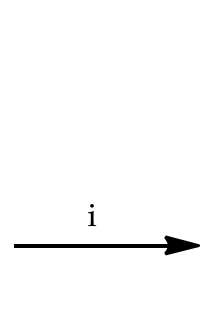<smiles>[R]C1=NN(c2nc(C)c(C(=O)NN)s2)C([R])C1</smiles>

ii<smiles>Cc1nc(C)c(C(N)=O)s1</smiles>

iii<smiles>[R]C1=NN(C)C([R])C1</smiles>

$10 \quad 73 \%$

iv

a, $\mathrm{Ar}=\mathrm{C}_{6} \mathrm{H}_{5}$

$70-75 \%$

b, $\mathrm{Ar}=4-\mathrm{CH}_{3} \mathrm{C}_{6} \mathrm{H}_{4}$<smiles>[R]C1=NN(c2nc(C)c(-n3c(=O)[nH]c4ccccc4c3=O)s2)C([R])C1</smiles><smiles>[R]C1=NN(C(=S)SC)C([R])C1</smiles>

12

$66 \%$

$\mathrm{R}=$ 2-furanyl

iii $=\mathrm{C}_{6} \mathrm{H}_{5} \mathrm{NH}_{2}, 4-\mathrm{CH}_{3} \mathrm{C}_{6} \mathrm{H}_{4}$

$\mathrm{i}=\mathrm{H}_{2} \mathrm{NNH}_{2} \mathrm{H}_{2} \mathrm{O}$

iv = Anthranilic acid or methyl anthranilate

$\mathrm{ii}=\mathrm{NaNO}_{2} / \mathrm{HCl}$ $\mathrm{v}=$ 2-naphthol

Scheme 3 Synthesis of thiazole derivatives $(\mathbf{9}),(\mathbf{1 0})$, urea derivatives (11a and $\mathbf{1 1} \mathbf{b})$, quinazoline $\mathbf{1 2}$, and $\beta$-naphthyl carbamate (13)

7.65-7.68 (m, 2H, ArH's); ${ }^{13} \mathrm{C}-\mathrm{NMR}$ (DMSO-d6) $\delta 21.4$ $\left(\mathrm{CH}_{3}\right), 87.7,110.2,110.5,115.4,116.9,127.4,129.4$, $133.1,137.2,143,146.5,150.7,156.9,1159.1$; MS $(m / z)$ : $275\left(\mathrm{M}^{+}, 1\right), 274$ (9), 240 (43), 212 (19), 169 (34), 141 (35), 169 (34), 141 (35), 108 (28), 107 (21), 91 (9), 79 (31), 44 (100); Anal. Calcd. for $\mathrm{C}_{17} \mathrm{H}_{13} \mathrm{~N}_{3} \mathrm{O}$ (275.30): C, 74.17; $\mathrm{H}, 4.76$; N, 15.26; found: C, 74.21; H, 4.64; N, 15.15 .
2-Amino-6-(furan-2-yl)-4-(3-(furan-2-yl)-1-phenyl-1H-pyrazol-4-yl)nicotinonitrile (2b) Yellow solid from glacial acetic acid, yield (2.8 g, 72\%), mp: 183-184 ${ }^{\circ} \mathrm{C}$; IR $\left(\mathrm{KBr}, \mathrm{cm}^{-1}\right)$ : 3327, $3265\left(\mathrm{NH}_{2}\right), 3055$ $(=\mathrm{C}-\mathrm{H}), 2208(-\mathrm{CN}), 1647(-\mathrm{C}=\mathrm{N}){ }^{1} \mathrm{H}$ NMR $\left(\mathrm{CDCl}_{3}\right)$ : $\delta: 6.71\left(\mathrm{t}, 1 \mathrm{H}\right.$, furan $\left.\mathrm{H}-4^{\prime}\right), 7.14-7.16(\mathrm{~d}, 1 \mathrm{H}$, furan $\mathrm{H}-3)$, 7.48-7.96 $\left(\mathrm{m}, 12 \mathrm{H}, \mathrm{ArH}^{\prime}\right.$,, $\mathrm{NH}_{2}$, furan H's and pyridine 


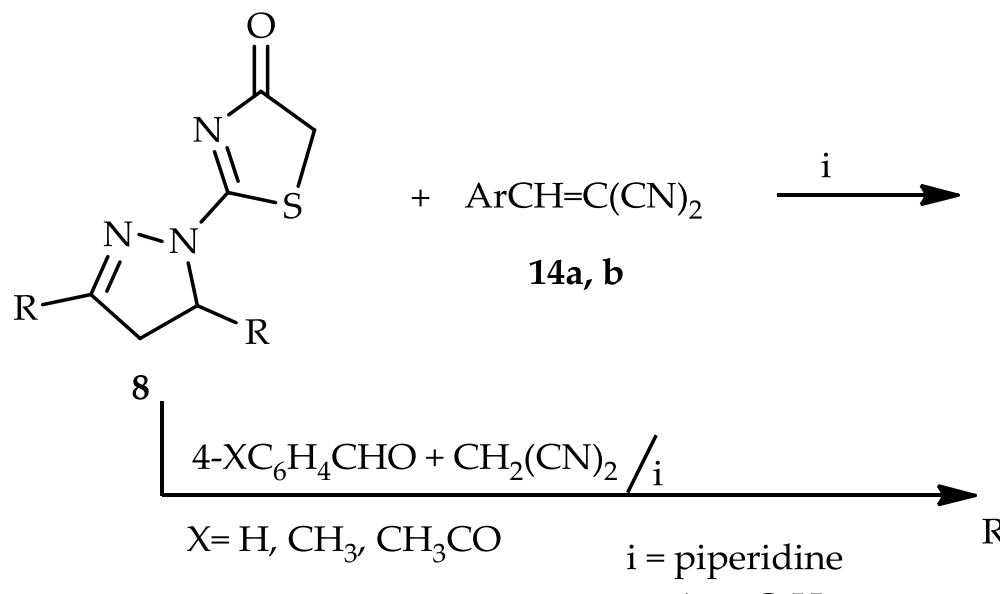

= piperidine

a, $\mathrm{Ar}=\mathrm{C}_{6} \mathrm{H}_{5}$

b, $\mathrm{Ar}=4-\mathrm{OCH}_{3} \mathrm{C}_{6} \mathrm{H}_{4}$
$\mathrm{H}_{2} \mathrm{~N}$<smiles>CC1=C(C#N)C(Br)c2sc(C)nc2O1</smiles>

Scheme 4 Synthesis of pyrano[2,3-d]thiazole derivatives (15a and $\mathbf{1 5} \mathbf{b})$

Table 1 Cytotoxicity $\left(\mathrm{IC}_{50}, \mu \mathrm{mol} \mathrm{mL}^{-1}\right.$ ) of the synthesized compounds (3a-11b) against MCF-7 and HCT-116 human cancer cell lines

\begin{tabular}{|c|c|c|c|c|c|}
\hline Compound no. & $\begin{array}{l}\text { MCF-7 } \\
\mathrm{IC}_{50}\left(\mu \mathrm{mol} \mathrm{mL} L^{-1}\right)\end{array}$ & $\begin{array}{l}\text { HCT-116 } \\
\mathrm{IC}_{50}\left(\mu \mathrm{mol} \mathrm{mL}{ }^{-1}\right)\end{array}$ & Compound no. & $\begin{array}{l}\text { MCF-7 } \\
\mathrm{IC}_{50}\left(\mu \mathrm{mol} \mathrm{mL} L^{-1}\right)\end{array}$ & $\begin{array}{l}\text { HCT-116 } \\
\mathrm{IC}_{50}\left(\mu \mathrm{mol} \mathrm{mL} \mathrm{m}^{-1}\right)\end{array}$ \\
\hline $3 a$ & 23.6 & 346 & 7 & 14.2 & $>500$ \\
\hline $4 a$ & 13.5 & 291 & 8 & 23.5 & $>500$ \\
\hline $4 d$ & 15.1 & 242 & 9 & 60.2 & 316 \\
\hline $4 e$ & 222 & 193 & $11 a$ & 203 & 215 \\
\hline $4 f$ & 238 & 124 & $11 b$ & 404 & 180 \\
\hline 5 & 9.65 & 213 & & & \\
\hline Imatinib & 24.5 & - & Imatinib & 24.5 & - \\
\hline Cisplatin & & 2.43 & Cisplatin & & 2.43 \\
\hline
\end{tabular}

$\mathrm{H}-5), 9.15$ (s, $1 \mathrm{H}$, pyrazole $\mathrm{H}-5) ;{ }^{13} \mathrm{C}-\mathrm{NMR}$ (DMSO-d6) $\delta: 90.1,112.0,112.1,114.1,114.3,115.2,116.9,117.6$, $120.3,127.5,128.3,129,5,137.4,140.8,141.3,141.7$, $143.5,144.7,148.7,150.2,159.4 ;$ MS $(\mathrm{m} / z): 393(\mathrm{M}+$, 1), 376 (7), 358 (10), 334 (1), 316 (24), 298 (40), 270 (17), 255 (24), 241 (14), 227 (16), 212 (13), 201 (15), 187 (16), 171 (14), 159 (17), 135 (20), 109 (20), 91 (22), 69 (23), 43 (100); Anal. Calcd. for $\mathrm{C}_{23} \mathrm{H}_{15} \mathrm{~N}_{5} \mathrm{O}_{2}$ (393.40): C, 70.22; H, 3.84; N, 17.80; found: C, 70.36; H, 3.84; N, 17.94 .

2-Amino-4-(3-(furan-2-yl)-1-phenyl-1H-pyrazol-4-yl)-6-(p-tolyl)nicotinonitrile (2c) Yellow solid from glacial acetic acid, yield (3.09 g, 74\%), mp: $200-203{ }^{\circ} \mathrm{C}$; IR $\left(\mathrm{KBr}, \mathrm{cm}^{-1}\right): 3307,3275\left(-\mathrm{NH}_{2}\right), 2924(-\mathrm{C}-\mathrm{H}), 2192$ $(-\mathrm{CN}) ;{ }^{1} \mathrm{H}$ NMR $\left(\mathrm{CDCl}_{3}\right): \delta: 2.44\left(\mathrm{~s}, 3 \mathrm{H}, 4-\mathrm{CH}_{3} \mathrm{C}_{6} \mathrm{H}_{4}\right)$, 5.22 (s, br., $\left.2 \mathrm{H}, \mathrm{NH}_{2}\right), 6.33-7.55(\mathrm{~m}, 13 \mathrm{H}, \mathrm{ArH}$ 's + furan H's + pyridine $\mathrm{H}-5), 9.45$ (s, $1 \mathrm{H}$, pyrazole $\mathrm{H}-5) ;{ }^{13} \mathrm{C}-\mathrm{NMR}$
(DMSO-d6) $\delta: 21.4\left(\mathrm{CH}_{3}\right), 91.6,112.1,113.5,115.5,116.9$, 117.6, 120.3, 127.6, 128.1, 129.3, 129.6, 131.3, 137.1, 138.0, 140.9, 141.3, 143.4, 150.2, 158.3, 158.6; MS $(\mathrm{m} / \mathrm{z}): 419$ (M+2, 4), $418(\mathrm{M}+1,23), 417(\mathrm{M}+, 100), 222(60), 195$ (70), 180 (48), 166 (6), 152 (8), 94 (6), 77 (2), 43 (15); Anal. Calcd. for $\mathrm{C}_{26} \mathrm{H}_{19} \mathrm{~N}_{5} \mathrm{O}$ (417.46): C, 74.80; $\mathrm{H}, 4.59 ; \mathrm{N}, 16.78$; found: C, 74.92; H, 4.70; N, 16.67 .

2-Amino-4-(1-phenyl-3-(p-tolyl)-1H-pyrazol-4-yl)-6-(p-tolyl)nicotinonitrile (2d) Yellow solid from benzene, yield (3.48 g, 79\%), mp: $225-227{ }^{\circ} \mathrm{C}$; IR $\left(\mathrm{KBr}, \mathrm{cm}^{-1}\right)$ : 3348, $3240\left(\mathrm{NH}_{2}\right), 3039(=\mathrm{C}-\mathrm{H}), 2920$ $(-\mathrm{C}-\mathrm{H}), 2214(-\mathrm{CN}) ;{ }^{1} \mathrm{H}$ NMR $\left(\mathrm{CDCl}_{3}\right): \delta: 2.39(\mathrm{~s}, 3 \mathrm{H}$, 4- $\left.\mathrm{CH}_{3} \mathrm{C}_{6} \mathrm{H}_{4}\right), 2.43$ (s, $\left.3 \mathrm{H}, 4-\mathrm{CH}_{3} \mathrm{C}_{6} \mathrm{H}_{4}\right), 5.22$ (s, br., $2 \mathrm{H}$, $\left.\mathrm{NH}_{2}\right), 7.24-7.82(\mathrm{~m}, 14 \mathrm{H}, \mathrm{ArH}$ 's + pyridine $\mathrm{H}-5), 8.40$ (s, $1 \mathrm{H}$, pyrazole $\mathrm{H}-5) ;{ }^{13} \mathrm{C}-\mathrm{NMR}$ (DMSO- $\left.d 6\right) \delta: 21.4\left(2 \mathrm{CH}_{3}\right)$, 91.7, 113.2, 115.2, 116.9, 120.3, 127.5, 127.7, 129.0, 129.3, 129.5, 129.6, 130.7, 133.1, 134.7, 136.2, 137.2, 137.4, 138.1, 
Table 2 Mean zone of inhibition beyond well diameter $(6 \mathrm{~mm})$ produced on a range of clinically pathogenic microorganisms using a $5 \mathrm{mg} \mathrm{mL}^{-1}$ concentration of tested samples

\begin{tabular}{|c|c|c|c|c|c|c|}
\hline Compound no. & $\begin{array}{l}\text { Aspergillus } \\
\text { fumigatus } \\
\text { (fungus) }\end{array}$ & $\begin{array}{l}\text { Candida } \\
\text { albicans } \\
\text { (fungus) }\end{array}$ & $\begin{array}{l}\text { Streptococcus } \\
\text { pneumonia (Gram + ve } \\
\text { bact.) }\end{array}$ & $\begin{array}{l}\text { Bacillus subtilis } \\
\text { (Gram + ve bact.) }\end{array}$ & $\begin{array}{l}\text { Pseudomonas } \\
\text { aeruginosa (Gram - ve } \\
\text { bact.) }\end{array}$ & $\begin{array}{l}\text { Escherichia } \\
\text { coli (Gram -ve } \\
\text { bact.) }\end{array}$ \\
\hline $2 a$ & 15.4 & 14.8 & 10.9 & 12.9 & 17.3 & 11.6 \\
\hline $2 b$ & 17.4 & 13.9 & 11.9 & 20.8 & 11.3 & 10.9 \\
\hline $2 e$ & 14.8 & 11.9 & 15.1 & 16.3 & 11.1 & 11.4 \\
\hline $2 f$ & 18.7 & 16.9 & 13.9 & 14.2 & 12.8 & 10.8 \\
\hline $3 a$ & 12.7 & 15.2 & 14.1 & 12.8 & 0 & 10.1 \\
\hline $3 b$ & 12.8 & 16.4 & 15.1 & 12.7 & 11.4 & 9.1 \\
\hline $3 d$ & 14.8 & 11.9 & 13.2 & 13.5 & 13.8 & 12.6 \\
\hline $3 e$ & 18.4 & 10.9 & 12.6 & 13.2 & 10.1 & 10.9 \\
\hline $3 f$ & 15.7 & 15.9 & 16.7 & 19.2 & 0 & 13.6 \\
\hline $4 a$ & 0.0 & 0.0 & 9.2 & 10.5 & 0 & 0 \\
\hline $4 b$ & 17.7 & 18.4 & 15.7 & 15.3 & 13.2 & 9.6 \\
\hline $4 c$ & 12.2 & 10.5 & 11.6 & 12.6 & 11.9 & 10.1 \\
\hline $4 e$ & 15.4 & 10.4 & 10.9 & 12.9 & 11.3 & 11.6 \\
\hline $4 f$ & 15.7 & 13.8 & 17.9 & 18.2 & 0 & 12.9 \\
\hline 6 & 16.2 & 12.5 & 16.8 & 14.6 & 12.1 & 12.8 \\
\hline $11 a$ & 19.1 & 16.9 & 13.6 & 14.7 & 12.1 & 10.4 \\
\hline $11 b$ & 14.8 & 16.3 & 15.1 & 16.3 & 11.1 & 11.4 \\
\hline 12 & 18.4 & 16.3 & 12.6 & 13.2 & 10.1 & 10.9 \\
\hline 13 & 20.8 & 16.8 & 13.1 & 10.8 & 13.4 & 12.3 \\
\hline Amphotericin B & 23.7 & 25.4 & - & - & - & - \\
\hline Ampicillin & - & - & 23.8 & 32.4 & - & - \\
\hline Gentamicin & - & - & - & - & 17.3 & 19.9 \\
\hline
\end{tabular}

Candida albicans and aspergillus fumigatus were resistant to compound $\mathbf{4 a}$

Pseudomonas aeruginosa was resistant to compounds $\mathbf{3 a}, \mathbf{3 f}, \mathbf{4 a}$, and $\mathbf{4 f}$

Aspergillus fumigatus was susceptible to compounds to $\mathbf{2 b}, \mathbf{2 f}, \mathbf{3 e}, \mathbf{4 b}, \mathbf{1 1} \mathbf{a}, \mathbf{1 2}$ and $\mathbf{1 3}$ while being moderate to $\mathbf{2 a}, \mathbf{2 e}, \mathbf{3 a}-\mathbf{3 d}, \mathbf{3 f}, \mathbf{4 c}, \mathbf{4 e - 4 f ,} \mathbf{6}$, and $\mathbf{1 1} \mathbf{b}$ when compared to the Amphotericin B standard

Candida albicans was moderate to all compounds except 4a when compared to the Amphotericin B standard

Streptococcus pneumoniae was moderate to all compounds when compared to the Ampicillin standard

Bacillus subtilis was moderate to all compounds when compared to the Ampicillin standard

Pseudomonas aeruginosa was moderate to all compounds except compounds $\mathbf{3 a}, \mathbf{3 f}, \mathbf{4 a}$, and $\mathbf{4 f}$, which were resistant to when compared to their standard Gentamicin Escherichia coli was moderate to all compounds except $\mathbf{4 a}$, which was resistant when compared to the Gentamicin standard

$141.3,149.8,158.3,158.7 ; \mathrm{MS}(\mathrm{m} / \mathrm{z}): 443(\mathrm{M}+2,0.51), 442$ $(\mathrm{M}+1,0.6), 441(\mathrm{M}+, 0.48), 426(31), 425(100), 411(6)$, 400 (6), 334 (10), 308 (3), 334 (10), 308 (3), 259 (8), 104 (16), 91 (30), 77 (94), 64 (42); Anal. Calcd. for $\mathrm{C}_{29} \mathrm{H}_{23} \mathrm{~N}_{5}$ (441.53): C, 78.89; H, 5.25; N, 15.86; found: C, 78.95; H, $5.18 ; \mathrm{N}, 15.63$.

2-Amino-4,6-di(furan-2-yl)nicotinonitrile (2e) Yellow solid from glacial acetic acid, yield (1.13 g, 45\%), mp: 213$215^{\circ} \mathrm{C}$; IR $\left(\mathrm{KBr}, \mathrm{cm}^{-1}\right)$ : 3374, $3298\left(\mathrm{NH}_{2}\right), 3008(=\mathrm{C}-\mathrm{H})$; ${ }^{1} \mathrm{H}$ NMR $\left(\mathrm{CDCl}_{3}\right)$ : $\delta: 6.24-6.27(\mathrm{t}, 1 \mathrm{H}$, furan $\mathrm{H}-4), 6.53-$ $6.54\left(\mathrm{t}, 1 \mathrm{H}\right.$, furan $\left.\mathrm{H}-4^{\prime}\right), 6.89-7.00(\mathrm{~d}, 1 \mathrm{H}$, furan $\mathrm{H}-2)$, 7.11-7.12 (d, $1 \mathrm{H}$, furan $\left.\mathrm{H}-5^{\prime}\right), 7.22(\mathrm{~s}, 1 \mathrm{H}$, pyridine $\mathrm{H}-4)$, 7.24-7.25 (d, $1 \mathrm{H}$, furan $\mathrm{H}-3), 7.40$ (s, br., $2 \mathrm{H}, \mathrm{NH}_{2}$ ), 8.10 (d, $1 \mathrm{H}$, furan $\mathrm{H}-5$ ); ${ }^{13} \mathrm{C}-\mathrm{NMR}$ (DMSO-d6) $\delta: 94.1,96.8,105.8$,
107.45, 114.6, 115.4, 115.7, 142.3, 143.4, 147.5, 151.3, 151.9, 152.9, 165.3. MS (m/z): $251(\mathrm{M}+, 3), 238(52), 181$ (23), 178 (86), 152 (19), 149 (23), 122 (18), 117 (15), 104 (27), 83 (44), 79 (16), 77 (18), 43 (100); Anal. Calcd. for $\mathrm{C}_{14} \mathrm{H}_{9} \mathrm{~N}_{3} \mathrm{O}_{2}$ (251.24): C, 66.93; H, 3.61; N, 16.73; found: C, 66.80; H, 3.72; N, 16.64 .

2-Amino-6-(furan-2-yl)-4-(1-phenyl-3-(p-tolyl)-1H-pyrazol-4-yl)nicotinonitrile (2f) Yellow solid from glacial acetic acid, yield $(2.75 \mathrm{~g}, 66 \%), \mathrm{mp}: 208-211{ }^{\circ} \mathrm{C}$; IR $(\mathrm{KBr}$, $\left.\mathrm{cm}^{-1}\right)$ : 3384, $3294\left(\mathrm{NH}_{2}\right), 2920(-\mathrm{C}-\mathrm{H}), 2200(-\mathrm{CN}), 1600$ $(-\mathrm{C}=\mathrm{N}) ;{ }^{1} \mathrm{H}$ NMR $\left(\mathrm{CDCl}_{3}\right): \delta: 2.30\left(\mathrm{~s}, 3 \mathrm{H}, 4-\mathrm{CH}_{3} \mathrm{C}_{6} \mathrm{H}_{4}\right)$, 6.27-6.28 (t, $1 \mathrm{H}$, furan $\mathrm{H}-4), 6.89-6.99(\mathrm{~d}, 1 \mathrm{H}$, furan $\mathrm{H}-3)$, $7.02(\mathrm{~s}, 1 \mathrm{H}$, pyridine $\mathrm{H}-5), 7.11-7.13(\mathrm{~d}, 1 \mathrm{H}$, furan $\mathrm{H}-2)$, 7.23-7.94 (m, $11 \mathrm{H}, \mathrm{ArH}$ 's $+\mathrm{NH}_{2}+$ furan- H's), $9.41(\mathrm{~s}, 1 \mathrm{H}$, 
pyrazole $\mathrm{H}-4) ;{ }^{13} \mathrm{C}-\mathrm{NMR}$ (DMSO-d6) $\delta: 21.4\left(\mathrm{CH}_{3}\right), 90.8$, 112.1, 114.3, 1146, 115.2, 120.3, 127.5, 129.0, 129.2, 129.5, 134.7, 136.4, 137.4, 141.2, 141.5, 144.5, 148.7, 149.8, 159.6; MS (m/z): $418(\mathrm{M}+1,23), 417(\mathrm{M}+, 100), 223(12), 222$ (60), 196 (98), 195 (70), 194 (15), 131 (38), 180 (48), 152 (8), 43 (15); Anal. Calcd. for $\mathrm{C}_{26} \mathrm{H}_{19} \mathrm{~N}_{5} \mathrm{O}$ (417.46): C, 74.80; $\mathrm{H}, 4.59 ; \mathrm{N}, 16.78$; found: C, 74.71; H, 4.65; N, 16.94 .

4-(Furan-2-yl)-2-phenyl-6-(p-tolyl)nicotinonitrile (3a) Yellow solid from glacial acetic acid, yield (2.15 g, 64\%), mp: $155-156{ }^{\circ} \mathrm{C}$; IR $\left(\mathrm{KBr}, \mathrm{cm}^{-1}\right): 3024(=\mathrm{C}-\mathrm{H})$, 3062, $2916(-\mathrm{C}-\mathrm{H}), 2214(-\mathrm{CN}) ;{ }^{1} \mathrm{H}$ NMR $\left(\mathrm{CDCl}_{3}\right): \delta: 2.44$ (s, 3H, 4- $\left.\underline{\mathrm{CH}}_{3} \mathrm{C}_{6} \mathrm{H}_{4}\right), 6.64-6.66(\mathrm{~d}, 1 \mathrm{H}$, furan $\mathrm{H}-4), 7.21(\mathrm{~s}$, $1 \mathrm{H}$, pyridine $\mathrm{H}-5), 7.27-7.83(\mathrm{~m}, 9 \mathrm{H}, \mathrm{ArH}$ 's and furan H-3, H-5), 8.44-8.46 (d, 2H, ArH's); ${ }^{13} \mathrm{C}-\mathrm{NMR}$ (DMSOd6) $\delta: 21.4\left(\mathrm{CH}_{3}\right), 106.8,110.3,113.5120 .3,125.6,126.4$, 127.5, 132.6, 138.3, 139.6, 142.5, 157.9, 171.7, 177.3, 183.9; MS $(\mathrm{m} / z): 337(\mathrm{M}+1,2), 336(\mathrm{M}+, 12), 245(6), 230(10)$, 202 (9), 180 (6), 158 (5), 132 (18), 65 (14); Anal. Calcd. for $\mathrm{C}_{23} \mathrm{H}_{16} \mathrm{~N}_{2} \mathrm{O}$ (336.39): C, 82.12; H, 4.79; N, 8.33; found: C, 82.00; H, 4.67; N, 8.45.

6-(Furan-2-yl)-4-(3-(furan-2-yl)-1-phenyl-1H-pyrazol-4-yl)-2-phenylnicotinonitrile (3b) White solid from glacial acetic acid, yield (3.22 g, 71\%), mp: $199-200{ }^{\circ} \mathrm{C}$; IR $\left(\mathrm{KBr}, \mathrm{cm}^{-1}\right)$ : $3052(=\mathrm{C}-\mathrm{H}), 2210(-\mathrm{CN}) ;{ }^{1} \mathrm{H}$ NMR $\left(\mathrm{CDCl}_{3}\right): \delta: 6.60-6.61(\mathrm{t}, 1 \mathrm{H}$, furan $\mathrm{H}-3), 6.77-6.81(\mathrm{~m}$, $3 \mathrm{H}$, furan H's), 7.12 (s, $1 \mathrm{H}$, pyridine $\mathrm{H}-5), 7.42-8.00$ (m, $12 \mathrm{H}, \mathrm{ArH}$ 's + furan-H's), $9.63(\mathrm{~s}, 1 \mathrm{H}$, pyrazole $\mathrm{H}-5) ;{ }^{13} \mathrm{C}-$ NMR (DMSO-d6) $\delta: 104.3,105.4,105.9,109.5,110.5$, $112.7,126.6,118.7,122.2,123.9,124.5,129.7,130.8,137.6$, 142.7, 140.6, 143.5, 149.8, 152.1, 153.6, 154.7, 163.7; MS $(\mathrm{m} / z): 455(\mathrm{M}+1,2), 454(\mathrm{M}+, 8), 382(16), 323(24), 262$ (93), 220 (55), 203 (19), 194 (41), 177 (21), 147 (31), 133 (52), 121 (37), 107 (56), 91 (16), 73 (66), 69 (100), 41 (42), 30 (49); Anal. Calcd. for $\mathrm{C}_{29} \mathrm{H}_{18} \mathrm{~N}_{4} \mathrm{O}_{2}$ (454.48): C, 76.64; $\mathrm{H}$, 3.99; N, 12.33; found: C, 76.52; H, 4.16; N, 12.28.

4-(3-(Furan-2-yl)-1-phenyl-1H-pyrazol-4-yl)-2-phenyl-6-(p-tolyl)nicotinonitrile (3c) White solid from glacial acetic acid, yield (3.59 g, 75\%), mp: $202-203{ }^{\circ} \mathrm{C}$; IR $\left(\mathrm{KBr}, \mathrm{cm}^{-1}\right)$ : $3040(=\mathrm{C}-\mathrm{H}), 2919(-\mathrm{C}-\mathrm{H}), 2213(-$ $\mathrm{CN}) ;{ }^{1} \mathrm{H}$ NMR $\left(\mathrm{CDCl}_{3}\right): \delta: 2.43\left(\mathrm{~s}, 3 \mathrm{H}, 4-\mathrm{CH}_{3} \mathrm{C}_{6} \mathrm{H}_{4}\right), 6.52$ $(\mathrm{t}, 1 \mathrm{H}$, furan $\mathrm{H}), 6.76(\mathrm{t}, 1 \mathrm{H}$, furan $\mathrm{H}), 7.16(\mathrm{~s}, 1 \mathrm{H}$, pyridine H-5), 7.27-8.07 (m, 15H, ArH's), 8.39 (s, 1H, pyyrazole $\mathrm{H}-5$ ); ${ }^{13} \mathrm{C}$-NMR (DMSO- $\left.d 6\right) \delta: 21.4\left(\mathrm{CH}_{3}\right), 100.2$, $104.4,112.4,115.3,118.6,121.1,122.2,123.8,124.3$, $126.4,129.7,130.7,136.6,137.9,139.7,142.1,142.8$, 149.7, 154.9, 160.5, 163.3; MS (m/z): $480(\mathrm{M}+1,4), 479$ (M+, 24), 478 (87), 449 (27), 321 (24), 304 (18), 277 (25), 249 (41), 322 (23), 219 (14), 205 (25), 179 (13), 166 (28), 152 (56), 29 (100); Anal. Calcd. for $\mathrm{C}_{32} \mathrm{H}_{22} \mathrm{~N}_{4} \mathrm{O}$ (478.54):
C, 80.32; H, 4.63; N, 11.71; found: C, 80.15; H, 4.50; N, 11.84 .

2-Phenyl-4-(1-phenyl-3-(p-tolyl)-1H-pyrazol-4-yl)-6-(p-tolyl)nicotinonitrile (3d) White solid from glacial acetic acid, yield (4.02 g, 80\%), mp: 216 $217^{\circ} \mathrm{C}$; IR $\left(\mathrm{KBr}, \mathrm{cm}^{-1}\right): 3033(=\mathrm{C}-\mathrm{H}), 2915(-\mathrm{C}-\mathrm{H}), 2211$ $(-\mathrm{CN}) ;{ }^{1} \mathrm{H} \mathrm{NMR}\left(\mathrm{CDCl}_{3}\right): \delta: 2.41\left(\mathrm{~s}, 3 \mathrm{H}, 4-\mathrm{CH}_{3} \mathrm{C}_{6} \mathrm{H}_{4}\right)$, $2.43\left(\mathrm{~s}, 3 \mathrm{H}, 4-\mathrm{CH}_{3} \mathrm{C}_{6} \mathrm{H}_{4}\right), 7.25$ (s, $1 \mathrm{H}$, pyridine $\left.\mathrm{H}-5\right)$, 7.22-8.03 (m, 18H, ArH's), $8.53(\mathrm{~s}, 1 \mathrm{H}$, pyrazole $\mathrm{H}-5)$; ${ }^{13} \mathrm{C}$-NMR (DMSO-d6) $\delta: 21.0\left(\mathrm{CH}_{3}\right), 21.4\left(\mathrm{CH}_{3}\right), 109.3$, $115.3,116.8,120.4,124.4,126.6,127.2,127.5,127.8$, $129.4,131.08,133.9,133.9,136.3,137.7,139.1,139.3$, $142.5,148.9,169.1,175.2,188.5$; MS $(m / z): 504(\mathrm{M}+2$, 0.5), $503(\mathrm{M}+1,2.7), 502(\mathrm{M}+, 7.7), 259$ (37), 251 (9), 234 (4), 214 (2), 79 (100), 77 (25), 65 (9), 63 (51), 60 (24), 57 (6); Anal. Calcd. for $\mathrm{C}_{35} \mathrm{H}_{26} \mathrm{~N}_{4}$ (502.61): C, 83.64; H, 5.21; N, 11.15; found: C, 83.52; H, 5.32; N, 11.06 .

4,6-Di(furan-2-yl)-2-phenylnicotinonitrile (3e) White solid from glacial acetic acid, yield (1.74 g, 56\%), mp: 213-214 ${ }^{\circ} \mathrm{C}$; IR $\left(\mathrm{KBr}, \mathrm{cm}^{-1}\right)$ : 3151; 3055 (=C-H), 2215 $(\mathrm{CN}) ;{ }^{1} \mathrm{H} \mathrm{NMR}\left(\mathrm{CDCl}_{3}\right): \delta: 6.74(\mathrm{t}, 1 \mathrm{H}$, furan $\mathrm{H}-3), 6.75$ (t, $1 \mathrm{H}$, furan $\left.\mathrm{H}-3^{\prime}\right), 7.30$ (s, $1 \mathrm{H}$, pyridine $\left.\mathrm{H}-5\right), 7.40-8.00$ (m, 7H, ArH's + furyl-H's), 8.10-8.12 (d, 2H, ArH's); ${ }^{13} \mathrm{C}-\mathrm{NMR}$ (DMSO-d6) $\delta$ : 101.6, 108.6, 109.5, 110.8, $112.0,121.4,126.5,126.9,134.8,141.3,142.6,143.5$, 156.7, 157.8, 171.6, 177.6, 197.7. MS $(m / z): 314(\mathrm{M}+2$, $0.2), 313$ (M 1, 1.7), $312(\mathrm{M}+, 100), 294$ (55), 299 (88), 239 (42), 223 (19), 210 (17), 197 (18), 179 (13), 167 (18), 110 (21), 81 (20), 55 (45), 41 (25); Anal. Calcd. for $\mathrm{C}_{20} \mathrm{H}_{12} \mathrm{~N}_{2} \mathrm{O}_{2}$ (312.32): C, 76.91; $\mathrm{H}, 3.87 ; \mathrm{N}, 8.97$; found: C, 76.83; H, 3.79; N, 9.12.

6-(Furan-2-yl)-2-phenyl-4-(1-phenyl-3-(p-tolyl)-1H-pyrazol-4-yl)nicotinonitrile (3f) White solid from glacial acetic acid, yield ( $2.39 \mathrm{~g}, 50 \%)$, mp: $186-187^{\circ} \mathrm{C}$; IR (KBr, $\left.\mathrm{cm}^{-1}\right)$ : 3056 (=C-H), $2917(-\mathrm{C}-\mathrm{H}), 2215(-\mathrm{CN}) ;{ }^{1} \mathrm{H}$ NMR $\left(\mathrm{CDCl}_{3}\right): \delta: 2.48\left(\mathrm{~s}, 3 \mathrm{H}, 4-\mathrm{CH}_{3} \mathrm{C}_{6} \mathrm{H}_{4}\right), 6.18-6.20(\mathrm{t}, 1 \mathrm{H}$, furan $\mathrm{H}-4), 6.88-6.89(\mathrm{~d}, 1 \mathrm{H}$, furan $\mathrm{H}-5), 7.9(\mathrm{~s}, 1 \mathrm{H}$, pyridine H-5), 7.31-7.85 (m, 13H, ArH's + furan-H's), 8.448.45 (d, 2H, ArH's), 9.24 (s, 1H, pyrazole $\mathrm{H}-5) ;{ }^{13} \mathrm{C}-\mathrm{NMR}$ (DMSO-d6) $\delta: 101.3,108.2,108.8,109.6,110.7,111.8$, 121.4, 126.6, 126.8, 134.7, 141.2, 142.5, 143.3, 131.8, 156.3, 158.2, 137.7, 171.5, 177.4, 180.1; MS $(m / z): 478(\mathrm{M}+, 5)$, 256 (10), 225 (12), 161 (12), 135 (19), 134 (12), 123 (14), 122 (100), 121 (73), 119 (11), 107 (13), 91 (19), 77 (10), 55 (17), 28 (17); Anal. Calcd. for $\mathrm{C}_{32} \mathrm{H}_{22} \mathrm{~N}_{4} \mathrm{O}$ (478.54): C, 80.32; H, 4.63; N, 11.71; found: C, 80.43; H, 4.54; N, 11.88 .

4-(Furan-2-yl)-2-oxo-6-(p-tolyl)-1,2-dihydropyridine-3-carbonitrile (4a) White solid from dioxane, yield $\left(2.62 \mathrm{~g}\right.$, 95\%), mp: $305-306{ }^{\circ} \mathrm{C}$; IR $\left(\mathrm{KBr}, \mathrm{cm}^{-1}\right): 3350$ 
(N-H), $3016(=\mathrm{C}-\mathrm{H}), 2912(-\mathrm{C}-\mathrm{H}), 2218(-\mathrm{CN}), 1654$ ($\mathrm{C}=\mathrm{O}) ;{ }^{1} \mathrm{H}$ NMR $\left(\mathrm{CDCl}_{3}\right): \delta: 2.38\left(\mathrm{~s}, 3 \mathrm{H}, 4-\mathrm{CH}_{3} \mathrm{C}_{6} \mathrm{H}_{4}\right), 6.83$ (t, 1H, Furyl H-5), 7.19 (s, 1H, pyridine $\mathrm{H}-5)$, 7.02-7.45 (m, 5H, ArH's + furyl-H's), 8.03-8.05 (d, 1H, furan H-5), $12.54(\mathrm{~s}, 1 \mathrm{H}, \mathrm{N}-\mathrm{H}) ;{ }^{13} \mathrm{C}-\mathrm{NMR}$ (DMSO-d6) $\delta: 21.2\left(\mathrm{CH}_{3}\right)$, 90.4, 120.2, 112.4, 115.7, 117.9, 126.3, 128.3, 134.3, 140.4, 142.6, 143.2, 146.4, 154.3, 158.4; MS (m/z): $278(\mathrm{M}+2,1)$, 277 (M+1, 15), 276 (M+, 100), 241 (9), 97 (55), 77 (20), 67 (24), 41 (8); Anal. Calcd. for $\mathrm{C}_{17} \mathrm{H}_{12} \mathrm{~N}_{2} \mathrm{O}_{2}$ (276.29): C, 73.90; H, 4.38; N, 10.14; found: C, 74.10; H, 4.52; N, 10.31 .

6-(Furan-2-yl)-4-(3-(furan-2-yl)-1-phenyl-1H-pyrazol-4-yl)-2-oxo-1,2-dihydropyridine-3-carbonitrile (4b) Yellow solid from glacial acetic acid, yield (3.47 g, 88\%), mp: $319-320{ }^{\circ} \mathrm{C}$; IR (KBr, cm $\left.{ }^{-1}\right): 3269(\mathrm{~N}-\mathrm{H}), 3123$ (=C-H), $2919(-\mathrm{C}-\mathrm{H}), 2216(-\mathrm{CN}), 1683(-\mathrm{C}=\mathrm{O}) ;{ }^{1} \mathrm{H}$ $\operatorname{NMR}\left(\mathrm{CDCl}_{3}\right): \delta: 6.53-6.59(\mathrm{t}, 1 \mathrm{H}$, furan $\mathrm{H}-4), 6.75-6.77$ (m, 2H, furan H-4', H-3), 7.38-7.79 (m, 8H, ArH's + furanH's), $8.22(\mathrm{~s}, 1 \mathrm{H}$, pyridine $\mathrm{H}-5), 8.38$ (s, $1 \mathrm{H}$, pyrazole $\mathrm{H}-=5$ ), 11.35 (s, $1 \mathrm{H}, \mathrm{NH}$ ); ${ }^{13} \mathrm{C}-\mathrm{NMR}$ (DMSO-d6) $\delta: 86.4$, 89.8, 105.0, 109.6, 111.1, 113.6, 118.9, 119.6, 123.2, 124.1, 126.2, 129.3, 134.5, 137.9, 139.2, 140.1, 144.6, 144.9, 145.2, 149.2, 156.9; MS (m/z): $395(\mathrm{M}+1,1), 394(\mathrm{M}+, 6), 393$ (49), 379 (29), 364 (8), 351 (8), 133 (9), 119 (11), 107 (33), 91 (100), 77 (8), 65 (19); Anal. Calcd. for $\mathrm{C}_{23} \mathrm{H}_{14} \mathrm{~N}_{4} \mathrm{O}_{3}$ (394.38): C, 70.05; H, 3.58; N, 14.21; found: C, 70.23; H, 3.50; N, 14.00 .

4 - (3 - (Furan-2 - yl) - 1 - phenyl-1H - pyrazol-4-yl)-2-oxo-6-(p-tolyl)-1,2-dihydropyridine-3-carbonitrile (4c) Pale yellow solid from dioxane, yield (3.89 g, 93\%), mp: 339-340 ${ }^{\circ} \mathrm{C}$; IR (KBr, cm $\left.{ }^{-1}\right): 3425(\mathrm{~N}-\mathrm{H}), 3105$ (=C-H), $2905(-\mathrm{C}-\mathrm{H}), 2214(-\mathrm{CN}), 1644(-\mathrm{C}=\mathrm{O}) ;{ }^{1} \mathrm{H}$ NMR $\left(\mathrm{CDCl}_{3}\right): \delta: 2.45\left(\mathrm{~s}, 3 \mathrm{H}, 4-\mathrm{CH}_{3} \mathrm{C}_{6} \mathrm{H}_{4}\right), 6.73(\mathrm{t}, 1 \mathrm{H}$, furan $\mathrm{H}-4)$, 6.67-6.68 (d, $1 \mathrm{H}$, furan $\mathrm{H}-3), 7.72-7.82(\mathrm{~m}$, $10 \mathrm{H}$, ArH's + furan $\mathrm{H}-5), 7.94(\mathrm{~s}, 1 \mathrm{H}$, pyridine $\mathrm{H}-5), 8.42$ (s, $1 \mathrm{H}$, pyrazole $\mathrm{H}-5), 11.61(\mathrm{~s}, 1 \mathrm{H}, \mathrm{NH}) ;) ;{ }^{13} \mathrm{C}-\mathrm{NMR}$

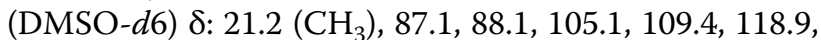
120.3, 123.3, 124.4, 124.8, 127.3, 129.2, 136.8, 137.8, 137.8, $139.4,140.2,145.5,149.2,157.9,163.5 ; \mathrm{MS}(\mathrm{m} / z): 418$ (M+, 6), 280 (10), 256 (50), 245 (32), 163 (19), 120 (16), 91 (16), 61 (24), 43 (100), 31 (47), 15 (17); Anal. Calcd. for $\mathrm{C}_{26} \mathrm{H}_{18} \mathrm{~N}_{4} \mathrm{O}_{2}$ (418.45): C, 74.63; H, 4.34; N, 13.39; found: C, 74.50; H, 4.51; N, 13.61 .

$2-O x o-4-(1-p h$ e nyl-3-(p-tolyl)- 1 H-pyrazol-4-yl)-6-(p-tolyl)-1,2-dihydropyridine-3-carbonitrile (4d) White solid from glacial acetic acid, yield (3.76 g, 85\%), mp: $325-326{ }^{\circ} \mathrm{C}$; IR (KBr, cm $\left.{ }^{-1}\right)$ : 3441 (N-H), 3131 (=C-H aromatic), $3016(=\mathrm{C}-\mathrm{H}), 2914(-\mathrm{C}-\mathrm{H}), 2215$ $(-\mathrm{CN}), 1640(-\mathrm{C}=\mathrm{O}) ;{ }^{1} \mathrm{H}$ NMR $\left(\mathrm{CDCl}_{3}\right): \delta: 2.40(\mathrm{~s}, 3 \mathrm{H}$, 4- $\left.\underline{\mathrm{CH}}_{3} \mathrm{C}_{6} \mathrm{H}_{4}\right), 2.45$ (s, 3H, 4- $\left.\underline{\mathrm{CH}}_{3} \mathrm{C}_{6} \mathrm{H}_{4}\right), 7.27-7.46$ (m, 10 $\mathrm{H}$, ArH's), 7.64-7.97 (m, 4H, ArH's and pyridine H-5),
9.23 (s, $1 \mathrm{H}$, pyrazole $\mathrm{H}-5), 11.61(\mathrm{~s}, 1 \mathrm{H}, \mathrm{NH}) ;{ }^{13} \mathrm{C}-\mathrm{NMR}$

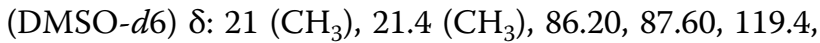
123.6, 127.5, 127.7, 128.4, 129.2,129.7, 136.6, 139.5, 140.6, 144.5, 150.3,150.8, 157.9, 164.1; MS ( $\mathrm{m} / z): 443(\mathrm{M}+1,5)$, 442 (M+, 28), 441 (28), 424 (14), 415 (100), 397 (7), 295 (5), 268 (4), 199 (7), 191 (5), 140 (4), 118 (16), 104 (8), 91 (24), 77 (55), 63 (25), 51 (12); Anal. Calcd. for $\mathrm{C}_{29} \mathrm{H}_{22} \mathrm{~N}_{4} \mathrm{O}$ (442.51): C, 78.71; H, 5.01; N, 12.66; found: C, 78.66; H, $5.18 ; \mathrm{N}, 12.77$.

4,6-Di(furan-2-yl)-2-oxo-1,2-dihydropyridine-3-carbonitrile (4e) White solid from dioxane, yield (1.38 g, 55\%), mp: $342-343{ }^{\circ} \mathrm{C}$; IR $\left(\mathrm{KBr}, \mathrm{cm}^{-1}\right): 3445(\mathrm{~N}-\mathrm{H}), 3115$ $(=\mathrm{C}-\mathrm{H}), 2216(-\mathrm{CN}), 1640-\mathrm{C}=\mathrm{O}) ;{ }^{1} \mathrm{H}$ NMR $\left(\mathrm{CDCl}_{3}\right)$ : $\delta$ : 6.66-6.68 (t, $1 \mathrm{H}$, furan $\mathrm{H}-4), 6.72(\mathrm{~d}, 1 \mathrm{H}$, furan $\mathrm{H}-3)$, 6.82-6.84 ( $\mathrm{t}, 1 \mathrm{H}$, furan $\left.\mathrm{H}-3^{\prime}\right)$, 7.16-7.25 $(\mathrm{m}, 4 \mathrm{H}$, furan H's + pyridine $\mathrm{H}-5$, furan H's), $11.63(\mathrm{~s}, 1 \mathrm{H}, \mathrm{N}-\mathrm{H}) ;{ }^{13} \mathrm{C}-$ NMR (DMSO-d6) $\delta: 14.0,58.6,98.8,102.5,103.6 .106 .8$, 115.6, 120.3, 141.9, 142.5, 143.4, 143.9, 151.3, 156.8, 159.7, 196.8. MS (m/z): $252(\mathrm{M}+, 4), 249$ (16), 245 (16), 218 (13), 203 (11), 184 (17), 173 (18), 171 (91), 156 (29), 155 (14), 144 (18), 129 (35), 115 (26), 91 (14), 28 (100); Anal. Calcd. for $\mathrm{C}_{14} \mathrm{H}_{8} \mathrm{~N}_{2} \mathrm{O}_{3}$ (252.22): C, 66.67; H, 3.20; N, 11.11; found: C, 66.78; H, 3.00; N, 11.25.

6-(Furan-2-yl)-2-oxo-4-(1-phenyl-3-(p-tolyl)-1H-pyrazol-4-yl)-1,2-dihydropyridine-3-carbonitrile (4f) Pale yellow solid from dioxane, yield (3.76 g, 90\%), mp: $311-$ $31{ }^{\circ} \mathrm{C}$; IR (KBr, cm $\left.{ }^{-1}\right)$ : $3421(\mathrm{~N}-\mathrm{H}), 3118$ (=C-H), 2911 $(-\mathrm{C}-\mathrm{H}), 2213(-\mathrm{CN}), 1648(-\mathrm{C}=\mathrm{O}) ;{ }^{1} \mathrm{H}$ NMR $\left(\mathrm{CDCl}_{3}\right)$ : $\delta: 2.50\left(\mathrm{~s}, 3 \mathrm{H}, 4-\mathrm{CH}_{3} \mathrm{C}_{6} \mathrm{H}_{4}\right), 6.63-6.65(\mathrm{t}, 1 \mathrm{H}$, furan $\mathrm{H}-4)$, 6.72-6.74 (d, 1H, furan H-3), 7.22-7.55 (m, 6H, ArH's and furan H-5), 7.79-7.81 (d, 2H, ArH's), 8.03-8.05 (d, 2H, ArH,s), 8.22 (s, $1 \mathrm{H}$, pyridine $\mathrm{H}-5), 8.35$ (s, $1 \mathrm{H}$, pyrazole $\mathrm{H}-5), 11.62$ (s, 1H, NH);); ${ }^{13} \mathrm{C}-\mathrm{NMR}$ (DMSO-d6) $\delta: 21$ (CH3), 87.2, 89.4, 110.6, 113.4, 119.5, 123.5, 127.3, 127.6, 129.2, 129.4, 129.6, 139.3, 139.6, 143.2, 144.5, 145.2, 150.2, 150.6, 156.6; MS (m/z): $418(\mathrm{M}+, 2), 417$ (100), 223 (12), 222 (60), 195 (70), 194 (15), 181 (38), 180 (48), 43 (15); Anal. Calcd. for $\mathrm{C}_{26} \mathrm{H}_{18} \mathrm{~N}_{4} \mathrm{O}_{2}$ (418.45): C, 74.63; H, 4.34; N, 13.39; found: C, 74.84; H, 4.21; N, 13.50 .

\section{3,5-Di(furan-2-yl)-4,5-dihydro-1H-pyrazole-1-carbothioam- ide (5), Mp: $164-166^{\circ} \mathrm{C}$ (lit. mp: $162-163^{\circ} \mathrm{C}$ ) [35]}

Ethyl 2-(3,5-di(furan-2-yl)-4,5-dihydro-1H-pyrazol-1-yl)-4-methylthiazole-5-carboxylate (6) A mixture of 3,5-di(furan-2-yl)-4,5-dihydro- $1 H$-pyrazole-1-carbothioamide (5) (2.61 g, $10 \mathrm{mmol})$ and ethyl 2-chloroacetoacetate $(1.38 \mathrm{~mL}, 10 \mathrm{mmol})$ was heated under reflux in ethanolic triethylamine for $2 \mathrm{~h}$, then allowed to cool at room temperature. The precipitate formed was filtered off, and recrystallized from ethanol to obtain compound 
(6) as a yellow solid from ethanol, yield (3.15 g, 85\%), mp: 140-141 ${ }^{\circ} \mathrm{C}$; IR $\left(\mathrm{KBr}, \mathrm{cm}^{-1}\right): 3120(=\mathrm{C}-\mathrm{H}), 2979(-\mathrm{C}-\mathrm{H})$, $1735(\mathrm{C}=\mathrm{O}) ;{ }^{1} \mathrm{H}$ NMR $\left(\mathrm{CDCl}_{3}\right): \delta: 1.29\left(\mathrm{t}, 3 \mathrm{H}, \mathrm{CH}_{2} \mathrm{CH}_{3}\right)$, 2.54 (s, $3 \mathrm{H}, 4-\mathrm{CH}_{3}$-thiazole), 3.50 (dd, $1 \mathrm{H}$, pyrazoline- $\mathrm{H}$ ), $3.64(\mathrm{dd}, 1 \mathrm{H}$, pyrazoline- $\mathrm{H}), 4.21\left(\mathrm{q}, 2 \mathrm{H}, \mathrm{CH}_{2} \mathrm{CH}_{3}\right), 5.71$ (dd, $1 \mathrm{H}$, pyrazoline- $\mathrm{H}), 6.29-6.30(\mathrm{~d}, 1 \mathrm{H}$, furan $\mathrm{H}-4)$, 6.39-6.40 (t, $1 \mathrm{H}$, furan $\mathrm{H}-3), 6.52-6.55(\mathrm{t}, 1 \mathrm{H}$, furan $\mathrm{H}-4)$, 6.81-6.82 (d, $1 \mathrm{H}$, furan $\mathrm{H}-3), 7.32-7.33(\mathrm{~d}, 1 \mathrm{H}$, furan $\mathrm{H}-5), 7.55-7.57$ (d, $1 \mathrm{H}$, furan $\mathrm{H}-5) ;{ }^{13} \mathrm{C}-\mathrm{NMR}$ (DMSO-d6) $\delta: 14.3,15.9,30.2,41.2,59.9,60.9,96.8,104.7,105.0,105.5$, 110.1, 143.6, 144.9, 148.6, 149.7, 49.3, 156.5, 151.9, 164.9. MS $(m / z): 373(\mathrm{M}+2,3), 372(\mathrm{M}+1,23), 371(\mathrm{M}+, 86)$, 264 (11), 237 (100), 131 (42), 106 (16), 77 (26); Anal. Calcd. for $\mathrm{C}_{18} \mathrm{H}_{17} \mathrm{~N}_{3} \mathrm{O}_{4} \mathrm{~S}$ (371.41): C, 58.21; $\mathrm{H}, 4.61 ; \mathrm{N}, 11.31 ; \mathrm{S}$, 8.63; found: C, 58.33; H, 4.85; N, 11.16; S, 8.82.

1-(2-(3,5-Di(furan-2-yl)-4,5-dihydro-1 H-pyrazol-1-yl)-4-methylthiazol-5-yl)-ethanone (7) A mixture of 3,5-di(furan-2-yl)-4,5-dihydro- $1 H$-pyrazole-1-carbothioamide (5) (2.61 g, $10 \mathrm{mmol}$ ), and 3-chloro-2,4-pentanedione $(1.13 \mathrm{~mL}, 10 \mathrm{mmol})$ was heated under reflux in ethanolic triethylamine for $2 \mathrm{~h}$, then, allowed to cool at room temperature. The precipitate formed was filtered off, and recrystallized from glacial acetic acid to obtain compound (7) as a pale yellow solid from glacial acetic acid, yield (2.25 g, 66\%), mp: $149-151{ }^{\circ} \mathrm{C}$; IR $\left(\mathrm{KBr}, \mathrm{cm}^{-1}\right)$ : 3118 (=C-H aromatic), $2999(-\mathrm{C}-\mathrm{H}), 1695(\mathrm{C}=\mathrm{O}) ;{ }^{1} \mathrm{H}$ $\operatorname{NMR}\left(\mathrm{CDCl}_{3}\right): \delta: 2.41$ (s, 3H, $\underline{4-C H}_{3}$-thiazole), 2.55 (s, $\left.3 \mathrm{H},-\mathrm{COCH}_{3}\right), 3.52(\mathrm{dd}, 1 \mathrm{H}$, pyrazoline- $\mathrm{H}), 3.66(\mathrm{dd}, 1 \mathrm{H}$, pyrazoline-H), $5.72(\mathrm{dd}, 1 \mathrm{H}$, pyrazoline- $\mathrm{H}), 6.29-6.30(\mathrm{~d}$, $1 \mathrm{H}$, furan $\mathrm{H}-4), 6.39-6.40(\mathrm{t}, 1 \mathrm{H}$, furan $\mathrm{H}-3), 6.52-6.55(\mathrm{t}$, $1 \mathrm{H}$, furan $\mathrm{H}-4), 6.81-6.82(\mathrm{~d}, 1 \mathrm{H}$, furan $\mathrm{H}-3), 7.32-7.33$ (d, $1 \mathrm{H}$, furan $\mathrm{H}-5), 7.55-7.57(\mathrm{~d}, 1 \mathrm{H}$, furan $\mathrm{H}-5) ;{ }^{13} \mathrm{C}-$ NMR (DMSO-d6) $\delta: 17.1,28.6,41.2,59.9,104.6,105.0$, 105.6, 109.8, 127.3, 143.7, 177.7, 148.6, 149.2, 155.9, 156.6, 159.9, 189.9. MS $(\mathrm{m} / \mathrm{z}): 343(\mathrm{M}+2,3), 342(\mathrm{M}+1,22), 341$ (M+, 100), 240 (79), 176 (26), 148 (12), 132 (21), 130 (19), 118 (11), 77 (20), 29 (20); Anal. Calcd. for $\mathrm{C}_{17} \mathrm{H}_{15} \mathrm{~N}_{3} \mathrm{O}_{3} \mathrm{~S}$ (341.38): C, 59.81; H, 4.43; N, 12.31; S, 9.39; found: C, $59.78 ; \mathrm{H}, 4.25 ; \mathrm{N}, 12.11 ; \mathrm{S}, 9.48$.

2-(3,5-Di(furan-2-yl)-4,5-dihydro-1H-pyrazol-1-yl) thiazol-4(5H)-one (8) A mixture of 5-di(furan-2-yl)4,5-dihydro-1H-pyrazole-1-carbothioamide (5) (2.61 g, $10 \mathrm{mmol})$, and ethyl chloroacetate $(1.06 \mathrm{~mL}, 10 \mathrm{mmol})$ was heated under reflux in ethanolic triethylamine for $2 \mathrm{~h}$, before the reaction mixture was allowed to cool to room temperature. Next, the precipitate formed was filtered off, and recrystallized from dioxane to afford compound (8) as a white solid, yield (1.95 g, 65\%), mp: $242-245{ }^{\circ} \mathrm{C}$; IR $\left(\mathrm{KBr}, \mathrm{cm}^{-1}\right): 3150(=\mathrm{C}-\mathrm{H}$ aromatic), $2966(-\mathrm{C}-\mathrm{H}), 1694$ $(\mathrm{C}=\mathrm{O}) ;{ }^{1} \mathrm{H}$ NMR $\left(\mathrm{CDCl}_{3}\right): \delta: 3.67(\mathrm{dd}, 1 \mathrm{H}$, pyrazoline- $\mathrm{H})$, 3.87 (dd, $1 \mathrm{H}$, pyrazoline), 3.89 (s, $2 \mathrm{H}$, thiazolone), 5.88 (dd, $1 \mathrm{H}$, pyrazoline- $\mathrm{H}), 6.29-6.30(\mathrm{~d}, 1 \mathrm{H}$, furan $\mathrm{H}-4)$, 6.39-6.40 (t, $1 \mathrm{H}$, furan $\mathrm{H}-3)$, 6.52-6.55 (t, $1 \mathrm{H}$, furan $\mathrm{H}-4)$, 6.81-6.82 (d, $1 \mathrm{H}$, furan $\mathrm{H}-3), 7.32-7.33(\mathrm{~d}, 1 \mathrm{H}$, furan $\mathrm{H}-5)$, 7.55-7.57 (d, $1 \mathrm{H}$, furan $\mathrm{H}-5) ;{ }^{13} \mathrm{C}-\mathrm{NMR}$ (DMSOd6) $\delta: 37.6,41.1,61.3,104.7,105.0,105.6,111.3,143.7$, 177.6, 148.6, 149.2, 156.5, 159.8, 182.2. MS $(\mathrm{m} / \mathrm{z}): 301$ (M+, 3), 182 (20), 143 (11), 139 (21), 129 (17), 128 (10), 117 (27), 115 (39), 96 (16), 75 (19), 43 (100); Anal. Calcd. for $\mathrm{C}_{14} \mathrm{H}_{11} \mathrm{~N}_{3} \mathrm{O}_{3} \mathrm{~S}$ (301.32): C, 55.80; H, 3.68; N, 13.95; $\mathrm{S}$, 10.64; found: C, 55.70; H, 3.72; N, 14.18; S, 10.53 .

2-(3,5-Di(furan-2-yl)-4,5-dihydro-1H-pyrazol-1-yl)-4-methylthiazole-5-carbohydrazide (9) A mixture of ethyl 2-(3,5-di(furan-2-yl)-4,5-dihydro- $1 H$-pyrazol-1-yl)-4-methylthiazole-5-carboxylate (6) (3.71 g, $10 \mathrm{mmol}$ ) and $20 \mathrm{~mL}$ of hydrazine hydrate was heated under reflux for $12 \mathrm{~h}$, and the reaction mixture allowed to cool at room temperature. Next, the white precipitate was collected, washed with ethanol, and recrystallized from glacial acetic acid to afford compound (9); yield (2.32 g, 65\%), mp: $212-215^{\circ} \mathrm{C}$; IR $\left(\mathrm{KBr}, \mathrm{cm}^{-1}\right): 3430(\mathrm{~N}-\mathrm{H}), 3325$, $3273\left(\mathrm{NH}_{2}\right), 3076(=\mathrm{C}-\mathrm{H}), 2930(-\mathrm{C}-\mathrm{H}), 1646(\mathrm{C}=\mathrm{O})$; ${ }^{1} \mathrm{H}$ NMR $\left(\mathrm{CDCl}_{3}\right): \delta: 2.34\left(\mathrm{~s}, 3 \mathrm{H}, 4-\mathrm{CH}_{3}\right.$-thiazole), 3.41 (dd, $1 \mathrm{H}$, pyrazoline- $\mathrm{H}), 3.62(\mathrm{dd}, 1 \mathrm{H}$, pyrazoline- $\mathrm{H}), 5.59$ (dd, $1 \mathrm{H}$, pyrazoline- $\mathrm{H}), 6.29-7.64\left(\mathrm{~m}, 9 \mathrm{H}, \mathrm{N}-\mathrm{H}, \mathrm{NH}_{2}\right.$ and furan-H's); ${ }^{13} \mathrm{C}-\mathrm{NMR}$ (DMSO-d6) $\delta: 15.4,41.2,59.8$, 104.8, 105.0, 105.6, 109.2, 121.1, 143.6, 144.7, 148.7, 149.1, $156.3,156.8,161.2,164.8$. MS $(m / z): 358(\mathrm{M}+1,2), 357$ (M+, 11), 182 (16), 181 (100), 166 (36), 165 (11), 151 (38), 135 (24), 120 (17), 107 (29), 89 (16), 79 (32), 73 (38), 71 (11), 63 (11), 45 (91), 44 (12), 43 (38), 31 (14), 29 (16), 28 (23), 27 (16); Anal. Calcd. for $\mathrm{C}_{16} \mathrm{H}_{15} \mathrm{~N}_{5} \mathrm{O}_{3} \mathrm{~S}$ (357.39): C, 53.77; H, 4.23; N, 19.60; S, 8.97; found: C, 53.56; H, 4.34; N, 19.81; S, 9.17.

2-(3,5-Di(furan-2-yl)-4,5-dihydro-1H-pyrazol-1-yl)-4-methylthiazole-5-carbonyl azide (10) A sodium nitrite solution $(1.38 \mathrm{~g}, 20 \mathrm{mmol}$, water $(20 \mathrm{~mL}))$ was added portionwise to a suspension solution of 2-(3,5-di(furan-2-yl)-4,5-dihydro- $1 H$-pyrazol-1-yl)4-methylthiazole-5-carbohydrazide $(3.57 \mathrm{~g}, 10 \mathrm{mmol})$ in hydrochloric acid $(20 \mathrm{~mL}, 6 \mathrm{M})$ at $0-5{ }^{\circ} \mathrm{C}$ with stirring. A brownish yellow precipitate was formed, filtered off, washed with water, and recrystallized from water to afford compound (10) as a yellow color with yield (2.69 g, 73\%), mp: $162-164{ }^{\circ} \mathrm{C}$; IR $\left(\mathrm{KBr}, \mathrm{cm}^{-1}\right): 3133$ $(=\mathrm{C}-\mathrm{H}), 2927(-\mathrm{C}-\mathrm{H}), 2120\left(-\mathrm{N}_{3}\right), 1635(\mathrm{C}=\mathrm{O}) ;{ }^{1} \mathrm{H}$ $\operatorname{NMR}\left(\mathrm{CDCl}_{3}\right): \delta: 2.50\left(\mathrm{~s}, 3 \mathrm{H}, 4-\mathrm{CH}_{3}\right.$-thiazole), $3.40(\mathrm{dd}$, $1 \mathrm{H}$, pyrazoline- $\mathrm{H}), 3.83(\mathrm{dd}, 1 \mathrm{H}$, pyrazoline- $\mathrm{H}), 5.60(\mathrm{dd}$, $1 \mathrm{H}$, pyrazoline- $\mathrm{H}), 6.29-6.30(\mathrm{~d}, 1 \mathrm{H}$, furan $\mathrm{H}-4), 6.39-$ $6.40(\mathrm{t}, 1 \mathrm{H}$, furan $\mathrm{H}-3), 6.52-6.55(\mathrm{t}, 1 \mathrm{H}$, furan $\mathrm{H}-4)$, 6.81-6.82 (d, $1 \mathrm{H}$, furan $\mathrm{H}-3), 7.32-7.33(\mathrm{~d}, 1 \mathrm{H}$, furan $\mathrm{H}-5$ ), 7.55-7.57 (d, $1 \mathrm{H}$, furan $\mathrm{H}-5) ;{ }^{13} \mathrm{C}-\mathrm{NMR}$ (DMSO- 
d6) $\delta: 15.4,41.1,59.8,104.7,105.1,1.6 .2,109.3,111.5$, $143.7,144.6,148.5,149.8,156.4,158.9,161.4,165.0 ; M S$ $(\mathrm{m} / z): 369(\mathrm{M}+1,1), 368(\mathrm{M}+, 5), 327(12), 326(60), 311$ (19), 309 (19), 284 (23), 283 (14), 256 (17), 255 (100), 43 (14); Anal. Calcd. for $\mathrm{C}_{16} \mathrm{H}_{12} \mathrm{~N}_{6} \mathrm{O}_{3} \mathrm{~S}$ (368.37): C, 52.17; $\mathrm{H}, 3.28$; N, 22.81; S, 8.70; found: C, 52.34; H, 3.15; N, 22.67; S, 8.88.

\section{1-(Aryl)-4-methylthiazol-5-yl)-3-aryl urea}

(11a) and (11b) A mixture of 2-(3,5-di(furan-2-yl)-4,5-dihydro-1H-pyrazol-1-yl)-4-methylthiazole-5-carbonyl azide (10) (1.94 g, $5 \mathrm{mmol}$ ), and the appropriate aniline or 4-methylaniline ( $5 \mathrm{mmol}$ ), was heated under reflux in dioxane $(20 \mathrm{~mL})$ for $3 \mathrm{~h}$. The precipitate that formed after cooling at room temperature was collected, and recrystallized from dioxane.

1-(2-(3,5-Di(furan-2-yl)-4,5-dihydro-1H-pyrazol-1-yl)-4-methylthiazol-5-yl)-3-phenyl-urea (11a) Pale yellow solid from dioxane, yield (1.62 g, 75\%), mp: $191-192^{\circ} \mathrm{C}$; IR $\left(\mathrm{KBr}, \mathrm{cm}^{-1}\right): 3423(\mathrm{~N}-\mathrm{H}), 3035$ (=C-H aromatic), 2841 (-C-H), $1665(-\mathrm{CO}) ;{ }^{1} \mathrm{H}$ NMR $\left(\mathrm{CDCl}_{3}\right)$ : $\delta$ : 2.60 (s, $3 \mathrm{H}, 4-\mathrm{CH}_{3}$-thiazole), 3.49 (dd, $1 \mathrm{H}$, pyrazoline$\mathrm{H}), 3.88$ (dd, $1 \mathrm{H}$, pyrazoline-H), 5.89 (dd, $1 \mathrm{H}$, pyrazoline$\mathrm{H})$, 6.41-7.74 (m, 11H, ArH's + furan-H's), 10.72 (s, 2H, $2 \mathrm{~N}-\mathrm{H}$ ); ${ }^{13} \mathrm{C}-\mathrm{NMR}$ (DMSO-d6) $\delta: 11.5,41.6,59.9,104.5$, 105.3, 105.7, 106.4, 119.2, 121.7, 123.6, 125.5, 129.2, 138.3, 143.6, 144.3, 148.6, 149.3, 152.6, 156.6, 166.1; MS ( $\mathrm{m} / z)$ : 433 (M+, 1), 279 (12), 278 (75), 277 (44), 262 (20), 247 (10), 283 (17), 281 (24), 122 (10), 79 (14), 91 (14), 79 (14), 78 (17), 77 (27), 75 (19), 57 (23), 28 (100); Anal. Calcd. for $\mathrm{C}_{22} \mathrm{H}_{19} \mathrm{~N}_{5} \mathrm{O}_{3} \mathrm{~S}$ (433.48): C, 60.96; H, 4.42; N, 16.16; S, 7.40; found: C, 61.14; H, 4.28; N, 16.00; S, 7.45.

1 -(2-(3,5-Di(furan-2-yl)-4,5-dihydro-1H-pyrazol-1-yl)-4-methylthiazol-5-yl)-3-(p-tolyl)-urea (11b) White solid from dioxane, yield (1.56 g, 70\%), mp: $238-241{ }^{\circ} \mathrm{C}$; IR (KBr, cm $\left.{ }^{-1}\right)$ : $3432(\mathrm{~N}-\mathrm{H}), 3025$ (=C-H aromatic), $2914(-\mathrm{C}-\mathrm{H}), 1624(-\mathrm{C}=\mathrm{O}) ;{ }^{1} \mathrm{H}$ NMR $\left(\mathrm{CDCl}_{3}\right): \delta: 2.35\left(\mathrm{~s}, 3 \mathrm{H}, 4-\underline{\mathrm{CH}}_{3} \mathrm{C}_{6} \mathrm{H}_{4}\right), 2.50$ (s, 3H, 4- $\underline{\mathrm{CH}}_{3}-$ thiazole), 3.51 (dd, $1 \mathrm{H}$, pyrazoline- $\mathrm{H}), 3.88(\mathrm{dd}, 1 \mathrm{H}$, pyrazoline-H), 5.78 (dd, 1H, pyrazoline-H), 6.43-8.29 (m, $10 \mathrm{H}$, ArH's + furan-H's), 10.73 (s, 2H, $2 \mathrm{~N}-\mathrm{H}) ;{ }^{13} \mathrm{C}-\mathrm{NMR}$ (DMSO-d6) $\delta: 11.8,20.6,41.1,58.8,104.6,105.0,105.9$, 109.1, 121.6, 122.5, 125.4, 129.6, 131.9, 137.8, 143.7, 144.7, 148.5, 149.1, 151.8, 156.6, 165.8. MS $(\mathrm{m} / z): 447(\mathrm{M}+, 1)$, 411 (10), 380 (13), 232 (29), 191 (22), 190 (17), 189 (100), 162 (16), 134 (22), 43 (10); Anal. Calcd. for $\mathrm{C}_{23} \mathrm{H}_{21} \mathrm{~N}_{5} \mathrm{O}_{3} \mathrm{~S}$ (447.51): C, 61.73; H, 4.73; N, 15.65; S, 7.17; found: C, 61.76; H, 4.84; N, 15.72; S, 7.32.

3-(2-(3,5-Di(furan-2-yl)-4,5-dihydro-1H-pyrazol-1-yl)-4-methylthiazol-5-yl)quinazo-
line-2,4(1H,3H)-dione (12) Method A A mixture of 2-(3,5-di(furan-2-yl)-4,5-dihydro- $1 H$-pyrazol-1-yl)4-methylthiazole-5-carbonyl azide (10) (1.94 g, $5 \mathrm{mmol}$ ) and anthranilic acid (0.68 g, $5 \mathrm{mmol}$ ) was heated under reflux in dioxane $(20 \mathrm{~mL})$ for $4 \mathrm{~h}$. The solid that formed after the reaction mixture was cooled and recrystallized from glacial acetic acid to realize compound (12).

Method B A mixture of 2-(3,5-di(furan-2-yl)-4,5-dihydro-1H-pyrazol-1-yl)-4-methylthiazole-5-carbonyl azide (10) (1.94 g, $5 \mathrm{mmol})$ and methyl anthranilate $(0.75 \mathrm{~g}$, $5 \mathrm{mmol})$ was heated under reflux in dioxane $(20 \mathrm{~mL})$ for $4 \mathrm{~h}$. The solid that formed after the reaction mixture was cooled and recrystallized from glacial acetic acid produced a product identical in all respects $(\mathrm{mp}$, mixed $\mathrm{mp}$, and spectra) with compound (12). White solid from glacial acetic acid, yield (1.51 g, 66\%), mp: $161-162{ }^{\circ} \mathrm{C}$; IR (KBr, cm $\left.{ }^{-1}\right): 3415(\mathrm{~N}-\mathrm{H}), 3154$ (=C-H aromatic), 3046 (=C-H), $2950(-\mathrm{C}-\mathrm{H}), 1643(\mathrm{CO}) ;{ }^{1} \mathrm{H}$ NMR $\left(\mathrm{CDCl}_{3}\right): \delta$ : $2.34\left(\mathrm{~s}, 3 \mathrm{H}, 4-\mathrm{CH}_{3} \mathrm{C}_{6} \mathrm{H}_{4}\right), 3.43-3.52(\mathrm{dd}, 1 \mathrm{H}, J=12 \mathrm{~Hz}$, pyrazoline $\mathrm{CH}$ ), $3.81-3.90(\mathrm{dd}, 1 \mathrm{H}, J=12 \mathrm{~Hz}$, pyrazoline $\mathrm{CH}), 5.66-5.71(\mathrm{dd}, 1 \mathrm{H}, \mathrm{J}=12 \mathrm{~Hz}$, pyrazoline $\mathrm{CH})$, 6.12-8.17 (m, 10H, ArH's) and 10.6 (s, br., $1 \mathrm{H}, \mathrm{NH}) ;{ }^{13} \mathrm{C}-$

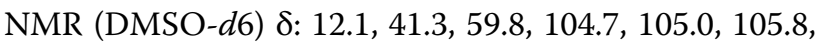
$109.1,114.9,117.2,123.2,114.9,126.9,135.4,136.2$, $139.8,143.6,144.6,147.1,148.6,149.2,159.5,157.4$, 163.8. MS (m/z): $459(\mathrm{M}+, 2), 300(8), 256(9), 256(11)$, 225 (12), 161 (12), 147 (32), 136 (20), 134 (13), 123 (15), 122 (100), 121 (74), 119 (11), 107 (14), 91 (20), 77 (10), 56 (10), 55 (17), 43 (12), 41 (10), 28 (17); Anal. Calcd. for $\mathrm{C}_{23} \mathrm{H}_{17} \mathrm{~N}_{5} \mathrm{O}_{4} \mathrm{~S}$ (459.48): C, 60.12; H, 3.73; N, 15.24; S, 6.98; found: $\mathrm{C}, 60.22 ; \mathrm{H}, 3.65 ; \mathrm{N}, 15.10 ; \mathrm{S}, 7.11$.

Naphthalen-2-yl(2-(3,5-di(furan-2-yl)-4,5-dihydro-1H-pyrazol-1-yl)-4-methylthiazol-5-yl)carbamate (13) A mixture of 2-(3,5-di(furan-2-yl)-4,5-dihydro$1 H$-pyrazol-1-yl)-4-methylthiazole-5-carbonyl azide (10) (1.94 g, $5 \mathrm{mmol}$ ) and 2-naphthol (0.72 g, $5 \mathrm{mmol})$, was heated under reflux in dry benzene $(20 \mathrm{~mL})$. The reaction mixture was allowed to cool at room temperature, then the precipitate that formed was collected and recrystallized from glacial acetic acid to afford compound (13) as a white solid from glacial acetic acid, yield (1.93 g, 80\%), mp: $225-227^{\circ} \mathrm{C}$; IR (KBr, cm $\left.{ }^{-1}\right): 3432(\mathrm{~N}-\mathrm{H}), 3115$ (=C-H aromatic), $2811(-\mathrm{C}-\mathrm{H}), 1603(\mathrm{C}=\mathrm{O}) ;{ }^{1} \mathrm{H}$ NMR $\left(\mathrm{CDCl}_{3}\right): \delta: 2.49\left(\mathrm{~s}, 3 \mathrm{H}, 4-\mathrm{CH}_{3}\right.$-thiazole), $3.34(\mathrm{dd}, 1 \mathrm{H}$, pyrazoline- $\mathrm{H}), 3.73$ (dd, $1 \mathrm{H}$, pyrazoline- $\mathrm{H}), 5.56$ (dd, $1 \mathrm{H}$, pyrazoline-H), 6.39-8.09 (m, 13H, ArH's + furan-H's), 10.2 (s, 1H, N-H); ${ }^{13} \mathrm{C}-\mathrm{NMR}$ (DMSO-d6) $\delta: ~ 11.5, ~ 41.6,59.9$, 105.3, 105.7, 109.2, 111.4, 113.3, 122.8, 128.7, 125.2, 125.6, 126.4, 128.9, 129.8, 134.7, 136.7, 143.5. 144.8, 148.4, 148.8, 156.8, 166.0; MS $(\mathrm{m} / z): 485(\mathrm{M}+1,1), 484(\mathrm{M}+, 3), 422$ (10), 403 (16), 402 (22), 360 (11), 319 (31), 318 (100), 275 (12), 274 (60), 273 (12), 225 (18), 121 (13), 85 (40), 57 (53), 
43 (11), 41 (11); Anal. Calcd. for $\mathrm{C}_{26} \mathrm{H}_{20} \mathrm{~N}_{4} \mathrm{O}_{4} \mathrm{~S}$ (484.53): C, 64.45; H, 4.16; N, 11.56; S, 6.62; found: C, 64.53; H, 4.23; N, 11.68; S, 6.77 .

\section{5-Amino-2-aryl-7-aryl'-7H-pyrano[2,3-d]thiazole-6-carbon- itrile $(15 a, b)$}

Method A A mixture of 2-(3,5-di(furan-2-yl)-4,5-dihydro-1H-pyrazol-1-yl)thiazol-4(5H)-one (1.5 g, $5 \mathrm{mmol})$, and the appropriate arylidene malononitrile (14a) or (14b) was heated under reflux in ethanol $(20 \mathrm{~mL})$ containing a catalytic amount of piperidine for $2 \mathrm{~h}$. The solid so formed after the reaction mixture was cooled to room temperature was collected and recrystallized from dioxane to yield compounds (15a, and $\mathbf{1 5 b})$, respectively.

Method B A mixture of 2-(3,5-di(furan-2-yl)-4,5-dihydro- $1 H$-pyrazol-1-yl)thiazol-4(5H)-one (1.5 g, $5 \mathrm{mmol})$, the appropriate amount of benzaldehyde or 4-methoxybenzaldehyde $(5 \mathrm{mmol})$, malononitrile $(0.33 \mathrm{~g}, 5 \mathrm{mmol})$ and piperidine $(0.42 \mathrm{~g}, 5 \mathrm{mmol})$ in $20 \mathrm{~mL}$ ethanol was heated under reflux for $2 \mathrm{~h}$. The solid that formed after the reaction mixture was cooled to room temperature was collected and recrystallized from dioxane to yield compounds identical in all aspects ( $\mathrm{mp}$, mixed $\mathrm{mp}$ and spectra) with the product obtained in method A.

5-Amino-2-(3,5-di(furan-2-yl)-4,5-dihydro-1H-pyrazol-1-yl)-7-phenyl-7H-pyrano[2,3-d]thiazole-6-carbonitrile (15a) Pale yellow solid from dioxane, yield (1.48 g, 65\%), mp: $295-296{ }^{\circ} \mathrm{C}$; IR $\left(\mathrm{KBr}, \mathrm{cm}^{-1}\right)$ : 3320, $3270\left(\mathrm{NH}_{2}\right), 3056(-\mathrm{C}-\mathrm{H}), 2988(-\mathrm{C}-\mathrm{H}), 2278(-\mathrm{CN})$; ${ }^{1} \mathrm{H}$ NMR $\left(\mathrm{CDCl}_{3}\right): \delta: 3.56(\mathrm{dd}, 1 \mathrm{H}$, pyrazoline- $\mathrm{H}), 4.02$ (dd, $1 \mathrm{H}$, pyrazoline- $\mathrm{H}), 5.11$ (dd, $1 \mathrm{H}$, pyrazoline- $\mathrm{H}), 5.50$ (s, $1 \mathrm{H}$, pyran $\mathrm{H}-4), 6.22\left(\mathrm{~s}, 2 \mathrm{H}, \mathrm{NH}_{2}\right), 6.80-7.63(\mathrm{~m}, 11 \mathrm{H}$, ArH's + furan-H's); ${ }^{13} \mathrm{C}-\mathrm{NMR}$ (DMSO-d6) $\delta: 34.1,38.2$, 59.9, 92.6, 104.8, 105.0, 109.1,125.7, 128.8, 129.1, 142.8, 143.3, 143.6, 144.7, 149.5, 149.3, 154.2, 155.4, 156.1, 156.6. MS ( $m / z): 456(\mathrm{M}+1,3), 455(\mathrm{M}+, 12), 382(17), 319(22)$, 318 (100), 290 (33), 151 (19), 128 (14); Anal. Calcd. for $\mathrm{C}_{24} \mathrm{H}_{17} \mathrm{~N}_{5} \mathrm{O}_{3} \mathrm{~S}$ (455.49): C, 63.29; H, 3.76; N, 15.38; S, 7.04; found: C, 63.38; H, 3.67; N, 15.16; S, 7.20.

5-Amino-2-(3,5-di(furan-2-yl)-4,5-dihydro-1H-pyrazol-1-yl)-7-(4-methoxyphenyl)-7H-pyrano[2,3-d]thiazole-6-carbonitrile (15b) Pale yellow solid from dioxane, yield (1.62 g, 67\%), mp: $304-307^{\circ} \mathrm{C}$; IR $\left(\mathrm{KBr}, \mathrm{cm}^{-1}\right)$ : 3320, $3273\left(\mathrm{NH}_{2}\right), 3070(-\mathrm{C}=\mathrm{H}), 2986(-\mathrm{C}-\mathrm{H}), 2228$ ($\mathrm{CN}) ;{ }^{1} \mathrm{H}$ NMR $\left(\mathrm{CDCl}_{3}\right): \delta: 3.52(\mathrm{dd}, 1 \mathrm{H}$, pyrazoline-H), $3.84\left(\mathrm{~s}, 3 \mathrm{H},-\mathrm{OCH}_{3}\right), 3.96(\mathrm{dd}, 1 \mathrm{H}$, pyrazoline- $\mathrm{H}), 5.12$ (dd, $1 \mathrm{H}$, pyrazoline- $\mathrm{H}), 5.55(\mathrm{~s}, 1 \mathrm{H}$, pyran- $\mathrm{H}), 6.22(\mathrm{~s}$, $2 \mathrm{H}, \mathrm{NH}_{2}$ ), 6.45-7.62 (m, 10H, ArH's + furan-H's); ${ }^{13} \mathrm{C}-$ NMR (DMSO-d6) $\delta: 34.3,36.5,41.3,56.2,59.8,92.7$, $104.5,105.7,106.1,116.4,131.5,134.8,142.8,143.6$, $144.8,148.4,148.9,154.2,155.2,155.7,156.3,165.5 ; \mathrm{MS}$ $(m / z): 485(\mathrm{M}+, 5), 478(24), 477(87), 446(25), 445$ (100), 399 (24), 396 (20), 373 (22), 372 (25), 327 (41), 326 (24), 251 (10); Anal. Calcd. for. for $\mathrm{C}_{25} \mathrm{H}_{19} \mathrm{~N}_{5} \mathrm{O}_{4} \mathrm{~S}$ (485.51): C, 61.85; H, 3.94; N, 14.42; S, 6.60; found: C, 61.73; H, 4.13; N, 14.35; S, 6.76.

\section{Evaluation of the antitumor activity using viability Assay}

Crystal violet stain (1\%), composed of $0.5 \%(\mathrm{w} / \mathrm{v})$ crystal violet and 50\% methanol, was made up to volume with $\mathrm{ddH}_{2} \mathrm{O}$ and filtered through a Whitman No. 1 filter paper.

\section{Cytotoxicity evaluation using viability assay}

Human hepatocellular breast (MCF-7) and colon (HCT116) carcinoma cells were obtained from the VACSERA Tissue Culture Unit. The cells were propagated in Dulbecco's modified Eagle's medium (DMEM), and supplemented with $10 \%$ heat-inactivated fetal bovine serum, $1 \% \mathrm{~L}$-glutamine, HEPES buffer and $50 \mu \mathrm{mol} \mathrm{mL}{ }^{-1}$ gentamycin. All cells were maintained at $37^{\circ} \mathrm{C}$ in a humidified atmosphere with $5 \% \mathrm{CO}_{2}$ and were sub-cultured twice a week.

\section{Evaluation of cytotoxicity activity}

Cytotoxicity of all compounds was tested in MCF-7 and HCT-116 cells. All experiments and data concerning the cytotoxicity evaluation were performed at the Regional Center for Mycology and Biotechnology RCMB, AlAzhar University, Cairo, Egypt. For the cytotoxicity assay, cells were seeded in a 96-well plate at a cell concentration of $1 \times 10^{4}$ cells per well in $100 \mu \mathrm{L}$ of growth medium. Fresh medium containing different concentrations of the test sample was added after $24 \mathrm{~h}$ of seeding. Serial two-fold dilutions of the tested compounds were added to confluent cell monolayers dispensed into 96-well, flat-bottomed microtiter plates (Falcon, NJ, USA) using a multichannel pipette. The microtiter plates were incubated at $37{ }^{\circ} \mathrm{C}$ in a humidified incubator with $5 \% \mathrm{CO}_{2}$ for a period of $48 \mathrm{~h}$. Three wells were used for each concentration of the test sample. Control cells were incubated without the test sample and with or without DMSO. The little percentage of DMSO present in the wells (maximal $0.1 \%$ ) was found not to affect the experiment. After incubation of the cells for at $37{ }^{\circ} \mathrm{C}$, various concentrations of the sample were added, and the incubation continued for $24 \mathrm{~h}$ before viable cell yield was determined using a colorimetric method. In brief, after the end of the incubation period, media were aspirated and the crystal violet solution (1\%) was added to each well for at least $30 \mathrm{~min}$. The stain was removed and the plates were rinsed using tap water until all excess stain was removed. Glacial acetic acid (30\%) was then added to all wells and mixed thoroughly, before the absorbance of the plates was measured (after being gently 
shaken) on a Microplate reader (TECAN, Inc.), using a test wavelength of $490 \mathrm{~nm}$. All results were corrected for background absorbance detected in wells without added stain. Treated samples were compared with the cell control in the absence of the tested compounds. All experiments were carried out in triplicate. The cell cytotoxic effect of each tested compound was calculated. Optical density was measured with a microplate reader (SunRise, TECAN, Inc., USA) to determine the number of viable cells, and the percentage of viability was calculated as the percentage of cell viability $=[1-(\mathrm{ODt} /$ ODc) $] \times 100 \%$ where ODt is the mean optical density of wells treated with the tested sample and ODc is the mean optical density of untreated cells. The relationship between the surviving cells and drug concentration was plotted to obtain the survival curve of each tumor cell line after treatment with the specified compound. The $50 \%$ inhibitory concentration $\left(\mathrm{IC}_{50}\right)$, the concentration required to cause toxic effects in $50 \%$ of intact cells, was estimated from graphic plots of the dose response curve for each concentration using Graphpad Prism software (San Diego, CA. USA).

\section{Antimicrobial activity assay}

Chemical compounds under investigation were individually tested against a panel of Gram-positive and Gram-negative bacterial pathogens, and fungi. Antimicrobial tests were conducted using the agar well-diffusion method [36-38]. After the media had cooled and solidified, wells ( $6 \mathrm{~mm}$ in diameter) were made in the solidified agar, before microbial inoculum was uniformly spread using a sterile cotton swab on a sterile Petri dish containing nutrient agar (NA) medium, or Sabouraud dextrose agar (SDA) media for bacteria and fungi, respectively. An amount of $100 \mu \mathrm{L}$ of the tested compound solution was prepared by dissolving $1 \mathrm{mg}$ of the compound in $1 \mathrm{~mL}$ of dimethylsulfoxide (DMSO). The inoculated plates were then incubated for $24 \mathrm{~h}$ at $37^{\circ} \mathrm{C}$ for bacteria and yeast, and $48 \mathrm{~h}$ at $28{ }^{\circ} \mathrm{C}$ for fungi. Negative controls were prepared using DMSO employed for dissolving the tested compound. Amphotericin B $(1 \mathrm{mg} / \mathrm{mL})$, Ampicillin $(1 \mathrm{mg} / \mathrm{mL})$, and Gentamicin $(1 \mathrm{mg} / \mathrm{mL})$ were used as standards for bacteria and fungi, respectively. After incubation, antimicrobial activity was evaluated by measuring the zone of inhibition against the tested microorganisms. Antimicrobial activity was expressed as inhibition diameter zones in millimeters $(\mathrm{mm})$.

\section{Conclusions}

In summary, new and efficient synthetic routes of some prepared pyridines, pyrazoline, thiazoles, and pyrano $[2,3-d]$ thiazole were achieved. The structure of the newly prepared compounds was established based on elemental analysis, spectral data, and alternative methods wherever possible. The synthesized compounds (3a, 4a, 4d-4f, 5, 7-9, 11a, and 11b) were investigated against two carcinoma cell lines: breast MCF-7 and colon HCT-116 human cancer cell lines. Our results showed that compounds $4 \mathbf{e}, \mathbf{4 f}, 11 \mathrm{a}$, and $\mathbf{1 1 b}$ had the lowest $\mathrm{IC}_{50}$ values against HCT-116 cancer cells. In addition, the selected newly prepared compounds were evaluated for their antimicrobial activity against Gram-positive and Gram-negative bacteria as well as some fungal-plants. The results proved that some prepared compounds showed an adequate inhibitory efficiency of growth of Gram-positive and Gram-negative bacteria.

\section{Abbreviations}

HCT-116: human cancer cell lines; MCF-7: estrogen responsive proliferative breast cancer model; DMEM: Dulbecco's modified Eagle's medium; HIV: human immunodeficiency virus; $I_{50}$ : the concentration of an inhibitor that is required for 50-percent inhibition of an enzyme in vitro; $\mathrm{mp}$ : melting point; Mw: molecular weight.

\section{Authors' contributions}

$\mathrm{AOA}, \mathrm{YHZ}$, and MSA designed the research, performed the research, analyzed the data, wrote the paper. All authors read and approved the final manuscript.

\section{Author details \\ ${ }^{1}$ Department of Chemistry, Faculty of Science, Beni-Suef University, Beni-Suef 62514, Egypt. ${ }^{2}$ Department of Chemistry, Faculty of Science and Humanity Studies at Al-Quwayiyah, Shaqra University, Al-Quwayi- yah 11971, Saudi Arabia. ${ }^{3}$ Department of Chemistry, Faculty of Science (Girls Branch), Al-Azhar University, Cairo, Egypt. ${ }^{4}$ Department of Chemistry, Faculty of Science, Cairo University, Giza 12613, Egypt.}

\section{Competing interests}

The authors declare that they have no competing interests.

Consent for publication

All Authors consent to the publication.

\section{Publisher's Note}

Springer Nature remains neutral with regard to jurisdictional claims in published maps and institutional affiliations.

Received: 1 December 2017 Accepted: 12 June 2018

Published online: 20 June 2018

References

1. Straub TS (1995) Epoxidation of a, $\beta$-unsaturated ketones with sodium perborate. Tetrahedron Lett 36(5):663-664

2. Sandler SR, Karo W (2013) Organic functional group preparations, 2nd edn. Elsevier, New York

3. Bergmann ED, Ginsburg D, Pappo R. (1959) The Michael reaction. Organic reactions

4. Prasad YR, Rao AL, Rambabu R (2008) Synthesis and antimicrobial activity of some chalcone derivatives. J Chem 5(3):461-466

5. Lopez SN, Castelli MV, Zacchino SA, Domínguez JN, Lobo G, CharrisCharris J, Cortés JC, Ribas JC, Devia C, Rodríguez AM (2001) In vitro antifungal evaluation and structure-activity relationships of a new series of chalcone derivatives and synthetic analogues, with inhibitory properties against polymers of the fungal cell wall. Bioorg Med Chem 9(8):1999-2013 
6. Baviskar B, Patel S, Baviskar B, Khadabadi S, Shiradkar M (2008) Design and synthesis of some novel chalcones as potent antimicrobial agent. Asian J Res Chem 1(2):67-69

7. Herencia F, Ferrandiz ML, Ubeda A, Domínguez J, Charris JE, Lobo GM, Alcaraz MJ (1998) Synthesis and anti-inflammatory activity of chalcone derivatives. Bioorg Med Chem Lett 8(10):1169-1174

8. Wu X, Wilairat P, Go M-L (2002) Antimalarial activity of ferrocenyl chalcones. Bioorg Med Chem Lett 12(17):2299-2302

9. Agarwal A, Srivastava K, Puri S, Chauhan PM (2005) Synthesis of 4-pyrido6-aryl-2-substituted amino pyrimidines as a new class of antimalarial agents. Bioorg Med Chem 13(22):6226-6232

10. Narender T, Khaliq T, Goyal N, Gupta S (2005) Synthesis of chromenochalcones and evaluation of their in vitro antileishmanial activity. Bioorg Med Chem 13(23):6543-6550

11. Cheng J-H, Hung C-F, Yang S-C, Wang J-P, Won S-J, Lin C-N (2008) Synthesis and cytotoxic, anti-inflammatory, and anti-oxidant activities of 2' 5'-dialkoxylchalcones as cancer chemopreventive agents. Bioorg Med Chem 16(15):7270-7276

12. Lin Y-M, Zhou Y, Flavin MT, Zhou L-M, Nie W, Chen F-C (2002) Chalcones and flavonoids as anti-tuberculosis agents. Bioorg Med Chem 10(8):2795-2802

13. Sivakumar P, Seenivasan SP, Kumar V, Doble M (2007) Synthesis, antimycobacterial activity evaluation, and QSAR studies of chalcone derivatives. Bioorg Med Chem Lett 17(6):1695-1700

14. Gibson M, Nguyen M, Zingales S (2017) Design, synthesis, and evaluation of (2-(Pyridinyl) methylene)-1-tetralone chalcones for anticancer and antimicrobial activity. Med Chem 14(4):333-343

15. Kocyigit UM, Budak Y, Gürdere MB, Ertürk F, Yencilek B, Taslimi P, Gülçin I, Ceylan M (2017) Synthesis of chalcone-imide derivatives and investigation of their anticancer and antimicrobial activities, carbonic anhydrase and acetylcholinesterase enzymes inhibition profiles. Arch Physiol Biochem 124:61-68

16. Patt WC, Hamilton HW, Taylor MD, Ryan MJ, Taylor DG Jr, Connolly CJ, Doherty AM, Klutchko SR, Sircar I (1992) Structure-activity relationships of a series of 2-amino-4-thiazole-containing renin inhibitors. J Med Chem 35(14):2562-2572

17. Sharma RN, Xavier FP, Vasu KK, Chaturvedi SC, Pancholi SS (2009) Synthesis of 4-benzyl-1,3-thiazole derivatives as potential anti-inflammatory agents: an analogue-based drug design approach. J Enzyme Inhib Med Chem 24(3):890-897

18. Jaen JC, Wise LD, Caprathe BW, Tecle H, Bergmeier S, Humblet CC, Heffner TG, Meltzer LT, Pugsley TA (1990) 4-(1,2,5,6-Tetrahydro-1-alkyl-3-pyridinyl)2-thiazolamines: a novel class of compounds with central dopamine agonist properties. J Med Chem 33(1):311-317

19. Tsuji K, Ishikawa H (1994) Synthesis and anti-pseudomonal activity of new 2-isocephems with a dihydroxypyridone moiety at C-7. Bioorg Med Chem Lett 4(13):1601-1606

20. Bell FW, Cantrell AS, Hoegberg M, Jaskunas SR, Johansson NG, Jordan $C L$, Kinnick MD, Lind P, Morin JM Jr (1995) Phenethylthiazolethiourea (PETT) compounds, a new class of HIV-1 reverse transcriptase inhibitors. 1. Synthesis and basic structure-activity relationship studies of PETT analogs. J Med Chem 38(25):4929-4936

21. Ergenc N Capan G, Günay NS, Oezkirimli S, Guengoer M, Özbey S, Kendi E (1999) Synthesis and hypnotic activity of new 4-thiazolidinone and 2-thioxo-4,5-imidazolidinedione derivatives. Arch Pharm 332(10):343-347

22. Carter JS, Kramer S, Talley JJ, Penning T, Collins P, Graneto MJ, Seibert K, Koboldt CM, Masferrer J, Zweifel B (1999) Synthesis and activity of sulfonamide-substituted 4,5-diaryl thiazoles as selective cyclooxygenase-2 inhibitors. Bioorg Med Chem Lett 9(8):1171-1174

23. Badorc A, Bordes M-F, de Cointet P, Savi P, Bernat A, Lalé A, Petitou M, Maffrand J-P, Herbert J-M (1997) New orally active non-peptide fibrinogen receptor (Gpllb-IIla) antagonists: identification of ethyl 3-[N-[4-[4-[amino [(ethoxycarbonyl) imino] methyl] phenyl]-1,3-thiazol2-yl]-N-[1-[(ethoxycarbonyl) methyl] piperid-4-yl] amino] propionate (SR 121787) as a potent and long-acting antithrombotic agent. J Med Chem 40(21):3393-3401
24. Rudolph J, Theis H, Hanke R, Endermann R, Johannsen L, Geschke F-U (2001) seco-Cyclothialidines: new concise synthesis, inhibitory activity toward bacterial and human DNA topoisomerases, and antibacterial properties. J Med Chem 44(4):619-626

25. Fares M, Abou-Seri SM, Abdel-Aziz HA, Abbas SE-S, Youssef MM, Eladwy RA (2014) Synthesis and antitumor activity of pyrido [2,3-d]pyrimidine and pyrido [2,3-d][1,2,4] triazolo[4,3-a]pyrimidine derivatives that induce apoptosis through $\mathrm{G} 1$ cell-cycle arrest. Eur J Med Chem 83:155-166

26. Malladi S, Anisetti R, Rao P (2014) A facile synthesis, in vitro antiinflammatory and antioxidant activity of novel benzimidazolylpyrano[2,3-d][1,3] thiazolocarbonitriles. Ind J Pharm Sci 76(6):510

27. Eldin SM (1999) Cyanothioacetamide and its derivatives in heterocyclic synthesis: a new route for the synthesis of several pyridine and thieno[2,3-b]pyridine derivatives and their biological evaluation. Zeitschrift für Naturforschung B 54(5):674-680

28. Abdelrazek FM, Michael FA, Mohamed AE (2006) Synthesis and molluscicidal activity of some 1,3,4-Triaryl-5-chloropyrazole, pyrano[2,3-c] pyrazole, pyrazolylphthalazine and pyrano[2,3-d]thiazole derivatives. Arch Pharm 339(6):305-312

29. Gomha SM, Kheder NA, Abdelhamid AO, Mabkhot YN (2016) One pot single step synthesis and biological evaluation of some novel bis (1,3,4-thiadiazole) derivatives as potential cytotoxic agents. Molecules 21(11):1532

30. Gomha SM, Ahmed SA, Abdelhamid AO (2015) Synthesis and cytotoxicity evaluation of some novel thiazoles, thiadiazoles, and pyrido[2,3-d] $[1,2,4]$ triazolo $[4,3-a]$ pyrimidin-5 $(1 \mathrm{H})$-ones incorporating triazole moiety. Molecules 20(1):1357-1376

31. Abdelhamid AO, Gomha SM, Abdelriheem NA, Kandeel SM (2016) Synthesis of new 3-heteroarylindoles as potential anticancer agents. Molecules 21(7):929

32. Zaki YH, Sayed AR, Elroby SA (2016) Regioselectivity of 1,3-dipolarcycloadditions andbantimicrobial activity of isoxazoline, pyrrolo[3,4-d]isoxazole4,6-diones, pyrazolo[3,4-d]pyridazines and pyrazolo[1,5-a]pyrimidines. Chem Cent J 10(17):1-13

33. Gomha SM, Zaki YH, Abdelhamid AO (2015) Utility of 3-Acetyl-6-bromo$2 \mathrm{H}$-chromen-2-one for the synthesis of new heterocycles as potential antiproliferative agents. Molecules 20(12):21826-21839

34. Zaki YH, Gomha SM, Mohamed AM (2017) Utility of 2-thioxo-pyrido [2,3-d] pyrimidinone in synthesis of pyridopyrimido[2,1-b][1,3,5]-thiadiazinones and pyridopyrimido [2,1-b][1,3]thiazinones as antimicrobial agents. Chem Cent J 11(1):57

35. Nasr MN, Said SA (2003) Novel 3,3a, 4,5,6,7-hexahydroindazole and arylthiazolylpyrazoline derivatives as anti-inflammatory agents. Arch Pharm 336(12):551-559

36. Sharma R, Sharma K, Dixit S (2010) Synthesis, characterization, and biological activities of some new arylazopyrazoles. Int J ChemTech Res 2:800-806

37. Studennikova L (1969) Hydrazones of acetaceric ester. Sb Nauch Ref Zh Kim 1(7173):46

38. Amir M, Agarwal R (1997) Synthesis and antibacterial activity of 1-thiocarbamoyl-3-methyl-4-(arylhydrazono)-2-pyrazolin-5-one. J Indian Chem Soc 74(2):154-155

\section{Submit your manuscript to a SpringerOpen ${ }^{\odot}$ journal and benefit from:}

- Convenient online submission

- Rigorous peer review

- Open access: articles freely available online

- High visibility within the field

- Retaining the copyright to your article

Submit your next manuscript at $\boldsymbol{\nabla}$ springeropen.com 